\title{
Role of Ultrasound in the Assessment of Female Infertility
}

\author{
${ }^{1}$ Maria Sheikh, ${ }^{2}$ Sanja Kupesic Plavsic
}

\begin{abstract}
Ultrasound has made significant advancements in reproductive medicine, especially infertility of a female cause. It is a useful tool in the diagnosis and management of various disorders. Transvaginal ultrasonography in particular plays a vital role in infertility treatment as it allows for evaluation of normal and stimulated ovarian cycles, aspiration of follicles, and subsequent transfer of embryos. The use of color Doppler permits visualization of endometrial and intraovarian vessels, facilitating an understanding of normal and abnormal physiology of the uterus and ovaries. This article reviews a variety of case scenarios regarding female infertility that may be encountered in the practice of reproductive endocrinology. Ovarian causes such as polycystic ovarian syndrome, luteinized unruptured follicle, luteal phase defect, premature ovarian failure, and endometriosis, are discussed together with tubal and uterine causes of infertility. By using illustrative images, the reader will be able to correlate findings on B-mode, color Doppler and 3D ultrasound with various causes of female infertility.
\end{abstract}

Keywords: Female infertility, Transvaginal ultrasound, Color Doppler, Polycystic ovary syndrome, Luteinized unruptured follicle syndrome, Luteal phase defect, Premature ovarian failure, Ovarian endometrioma, Pelvic inflammatory disease, Intrauterine adhesions, Septate uterus, Submucosal fibroid, Endometrial polyp.

How to cite this article: Sheikh M, Kupesic Plavsic S. Role of Ultrasound in the Assessment of Female Infertility. Donald School J Ultrasound Obstet Gynecol 2014;8(2):184-200.

\section{Source of support: Nil}

Conflict of interest: None

\section{INTRODUCTION}

Infertility is defined as failure of a couple to achieve pregnancy after 12 months of unprotected intercourse. It can have a significant psychological impact on those afflicted. Fortunately, many couples have favorable outcomes and end up conceiving without major interventions. For those who are not successful, there have been significant advancements in assisted reproductive technology (ART). Of the new methods, ultrasound has shown tremendous potential in the diagnosis and management of female infertility.

\footnotetext{
${ }^{1}$ Student Physician, ${ }^{2}$ Professor

1,2Department of Obstetrics and Gynecology, Paul L Foster School of Medicine, Texas Tech University at EI Paso, Texas, USA

Corresponding Author: Sanja Kupesic Plavsic, Assistant Academic Dean for Faculty Development; Professor of Obstetrics and Gynecology; Clinical Professor of Radiology, Department of Obstetrics and Gynecology, Paul L Foster School of Medicine Texas Tech University, 4801 Alberta Ave, El Paso, Texas 79905 USA, Phone: +1 (915)2156505, e-mail: sanja.kupesic@ttuhsc.edu
}

Transvaginal ultrasound is advantageous to transabdominal because it allows for better spatial resolution and avoids the need for a full urinary bladder. Two-dimensional (2D) B-mode ultrasound depicts sagittal and transverse planes. Three-dimensional (3D) ultrasound allows for sagittal, transverse, and coronal views giving a more detailed image of the pelvic organs. The addition of color Doppler via transvaginal probes allows visualization of the miniscule intraovarian and endometrial vessels. Deviations from normal structure and function can be detected with these modern methods of imaging.

There are numerous etiologies which factor into making a woman subfertile or infertile. Ovarian causes include polycystic ovary syndrome (PCOS), premature ovarian failure (POF), endometrioma, luteinized unruptured follicle (LUF) and luteal phase defect (LPD). Tubal factor infertility is most often associated with chronic pelvic inflammatory disease (PID). Uterine causes include submucosal fibroids, congenital Mullerian anomalies, such as septate uterus, endometrial polyps and intrauterine adhesions. The recent advances in ultrasound technique allow for the accurate diagnosis of these disorders. For some of these conditions, e.g. premature ovarian failure, ultrasonography has shown to be useful in formulating a treatment plan and predicting prognosis. The ten cases aforementioned are discussed here; ultrasound images are correlated with various clinical and laboratory findings to assist the reader in evaluation and management of female infertility.

\section{CASE REPORTS}

\section{Case 1}

A 32-year-old primary infertile female presents complaining of irregular menstrual periods since menarche which began at age 13. Her last menstrual period was three months ago. She also complains of acne and hair on her face, chin and abdomen. She denies changes in her voice, changes in vision, headaches and nipple discharge. Her past medical history is noncontributory. She states, she is not taking any medications and she is sexually active only with her husband. She is married for 5 years and has never conceived. On physical examination, she is markedly obese. Her BMI measures 32. She has visible facial and abdominal hair. On pelvic examination, the uterus is normal size and no adnexal masses are noted. 
Laboratory studies revealed negative beta-hCG, normal TSH and normal prolactin. Her FSH on day 3 of the menstrual cycle was normal; however, her LH was elevated. Her free testosterone was also elevated. Pelvic ultrasound was performed and findings are presented on Figures 1A to C.

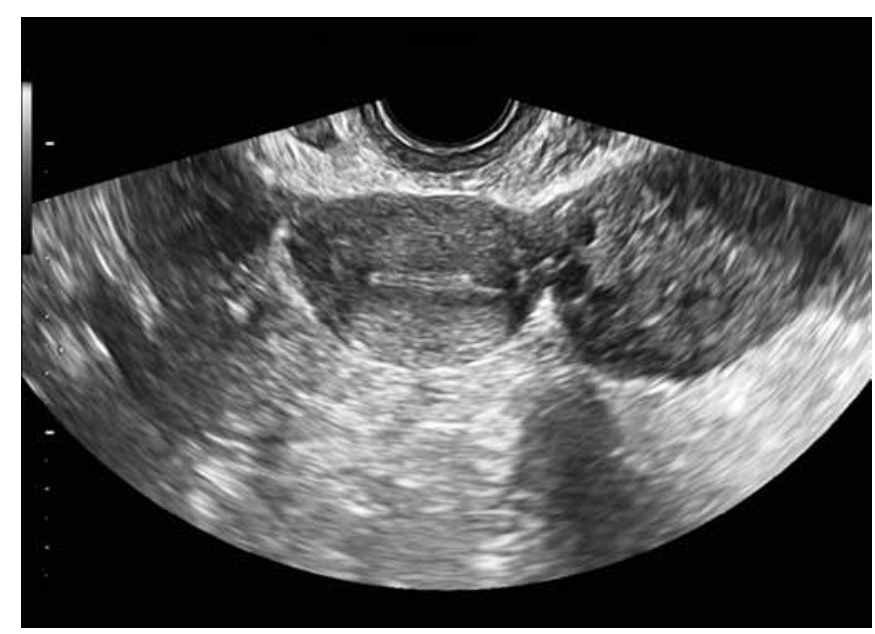

Fig. 1A: Transvaginal ultrasound of the patient from case 1. Note normal size uterus and enlarged ovary on the left

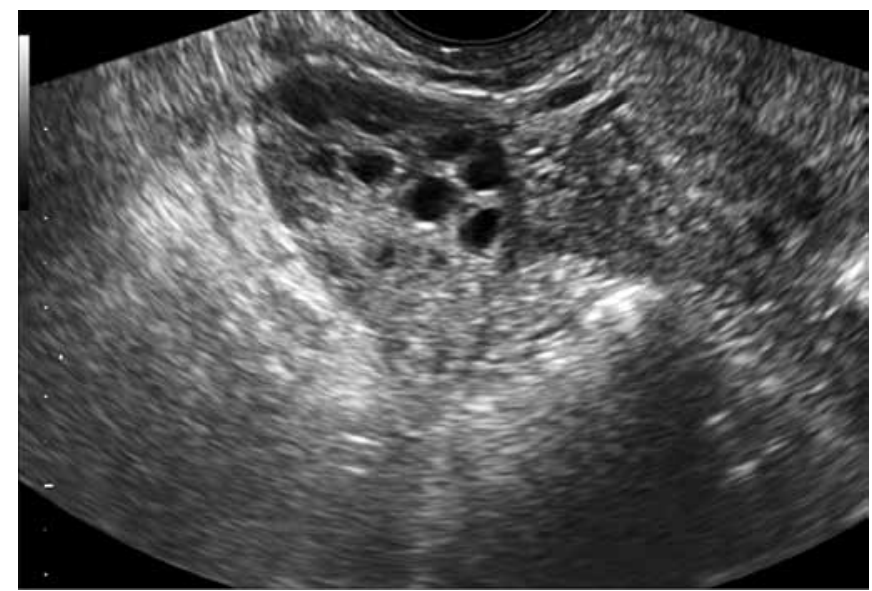

Fig. 1B: Transvaginal ultrasound reveals enlarged ovaries (volume of $15 \mathrm{cc}$ ). More than 12 follicles scattered throughout the enlarged ovarian stroma are clearly identified

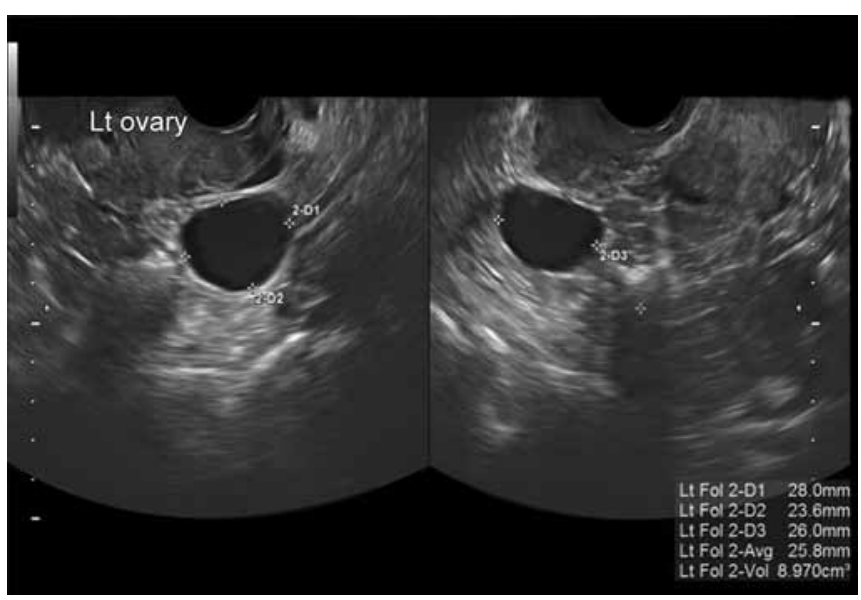

Fig. 2A: Transvaginal ultrasound reveals a unilocular cystic structure on the left ovary

\section{Case 2}

A 39-year-old primary infertile female presents with complaints of irregular menstrual bleeding for the past two years. She states that her periods have always been regular since menarche at age 14 but for the past two years, her cycles are usually prolonged and her periods are unpredictable. She denies any physical complaints. She is married for three years and her husband has two children from his previous marriage. On physical exam, she is afebrile and in no acute distress. Her vagina, cervix and uterus are within normal limits, and no adnexal masses are noted. Three months ago she started measuring her basal body temperature, which was typically biphasic. Elevation of the basal body temperature was noted on day 14 and remained high for the rest of her menstrual cycle.

Today is day 21 of the menstrual cycle; you decide to perform pelvic ultrasound.

A pelvic ultrasound reveals a $2.8 \times 2.6 \mathrm{~cm}$ cystic structure on the left ovary (Figs 2A and B) and a thickened secretory transformed endometrium. Color Doppler depicts peripheral

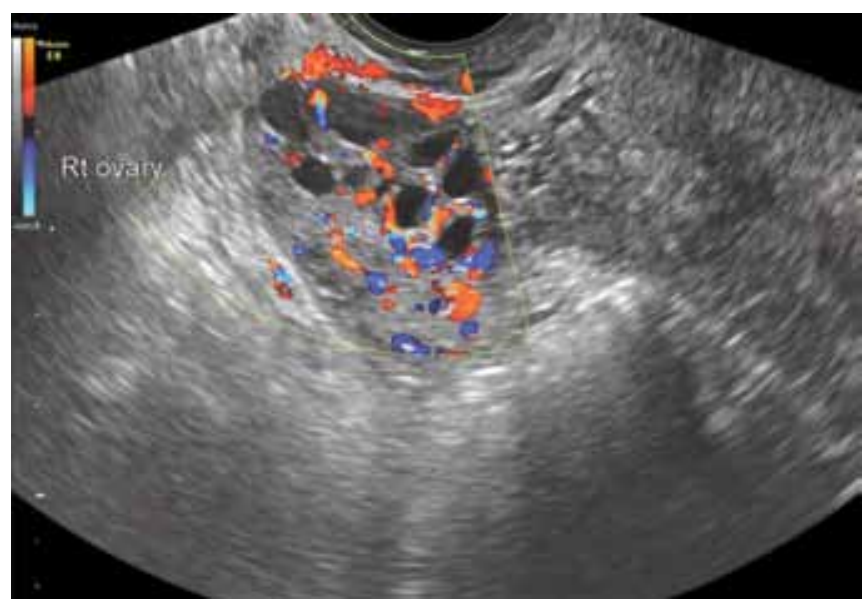

Fig. 1C: Color Doppler image of the ovary indicates increased intraovarian perfusion

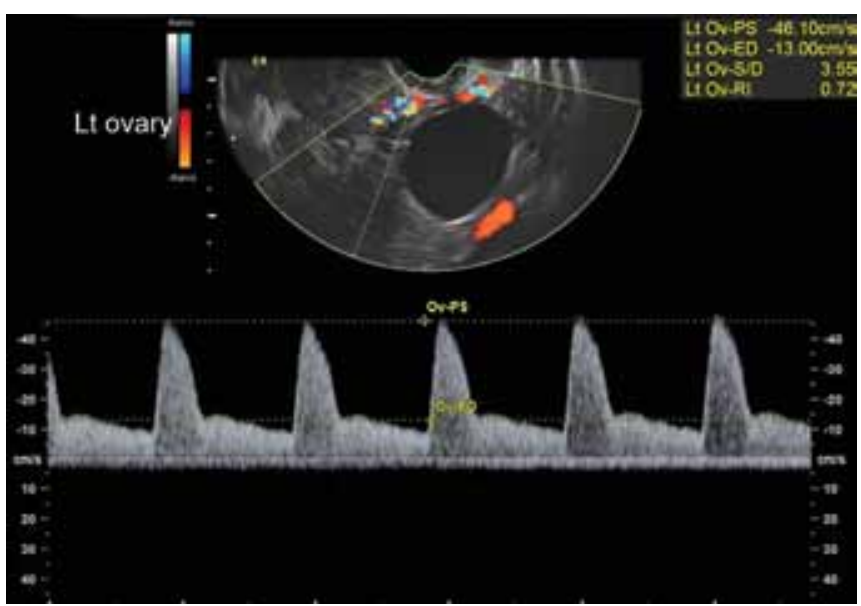

Fig. 2B: Color Doppler ultrasound of the same ovary. Moderate to high vascular impedance blood flow signals (RI 0.72) are obtained from the periphery of the cystic structure 
vessels with moderate to high vascular impedance (RI 0.72, PI 1.92). You decide to assess serum progesterone level. The result was normal for luteal phase and indicated ovulatory cycle.

\section{Case 3}

A 38-year-old G3P2012 patient presents with complaints of shortened menstrual cycles for the past year. Her history is significant for one spontaneous abortion 8 months ago at 7 weeks gestation. She states that her periods used to be regular since menarche at age 13 but for the past year, her cycles are short and occur every 20 to 23 days. She denies any physical complaints. She is married for seven years and has two children ( 5 and 3 years). On physical examination, she is afebrile and in no acute distress. Her pelvic examination was within normal limits. Today is the 20 th day of her menstrual cycle; you decide to order serum progesterone level and perform pelvic ultrasound.

A pelvic ultrasound reveals a normal size ovary with discrete luteal conversion blood flow signals (Fig. 3). Moderate impedance blood flow signals with resistive index of 0.54 were detected. Serum progesterone was significantly decreased $(1.8 \mathrm{ng} / \mathrm{ml})$, compared to normal values between 4.4 to $28.0 \mathrm{ng} / \mathrm{ml}$.

\section{Case 4}

A 27-year-old nulliparous primary infertile patient presents to your clinic with symptoms of amenorrhea and hot flashes. Her last menstrual period was 6 months ago. Her menarche was at 12 years, and she has had developed secondary sexual characteristics appropriately. Her menstrual cycles were always irregular, and she was taking oral contraception for 8 years (since her first coitus at age of 17). Thereafter her menstrual cycles were irregular and she experienced estrogen deficiency symptoms (vaginal dryness, hot flashes

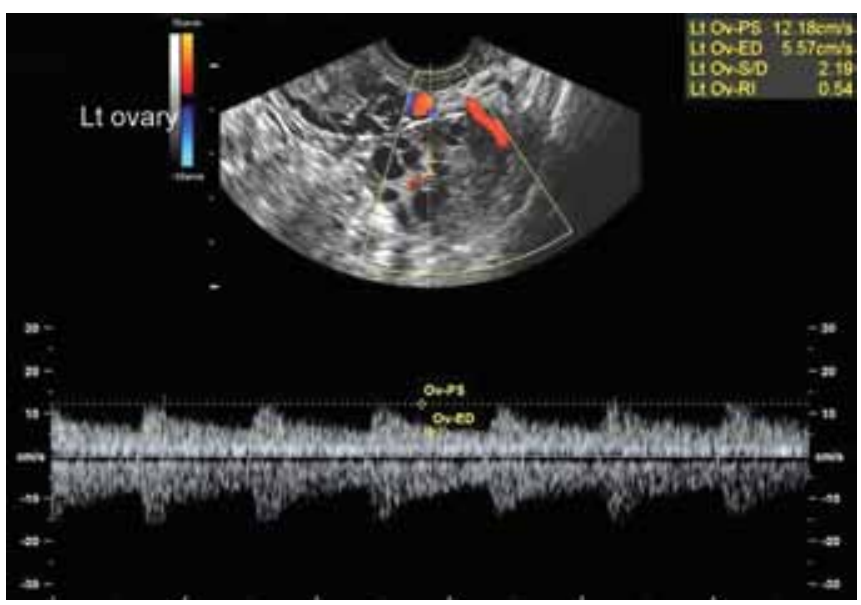

Fig. 3: Intraovarian blood flow during the mid-luteal phase in a patient from case 3 . Note increased resistance index $(R I=0.54)$ for luteal phase of the menstrual cycle and dyspareunia). Her serum gonadotropin concentrations were found to be elevated (FSH $40 \mathrm{mIU} / \mathrm{ml}$ and $\mathrm{LH} 55$ $\mathrm{mIU} / \mathrm{ml}$ ). Her pelvic ultrasound images are displayed on Figures $4 \mathrm{~A}$ to $\mathrm{C}$.

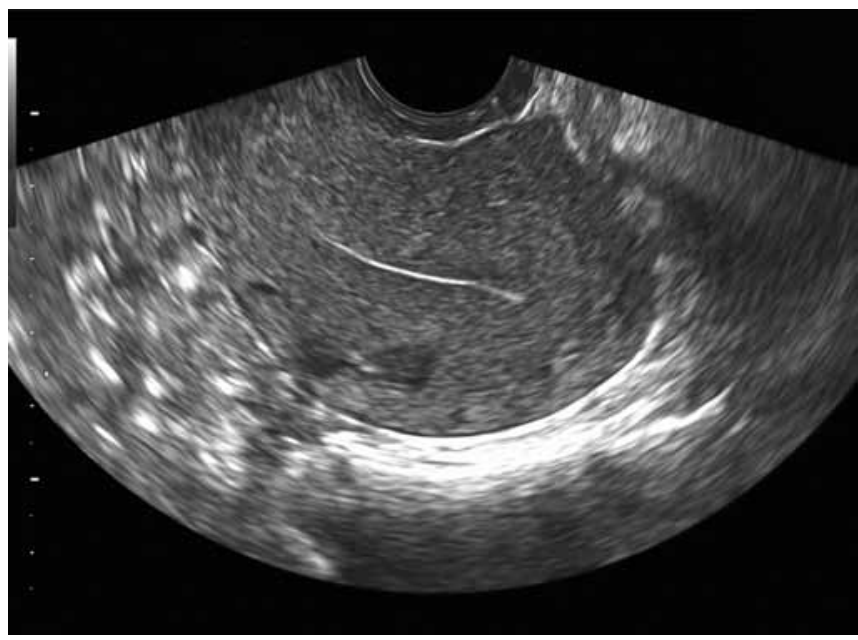

Fig. 4A: Transvaginal scan of the uterus. Note thin and hyperechogenic endometrium

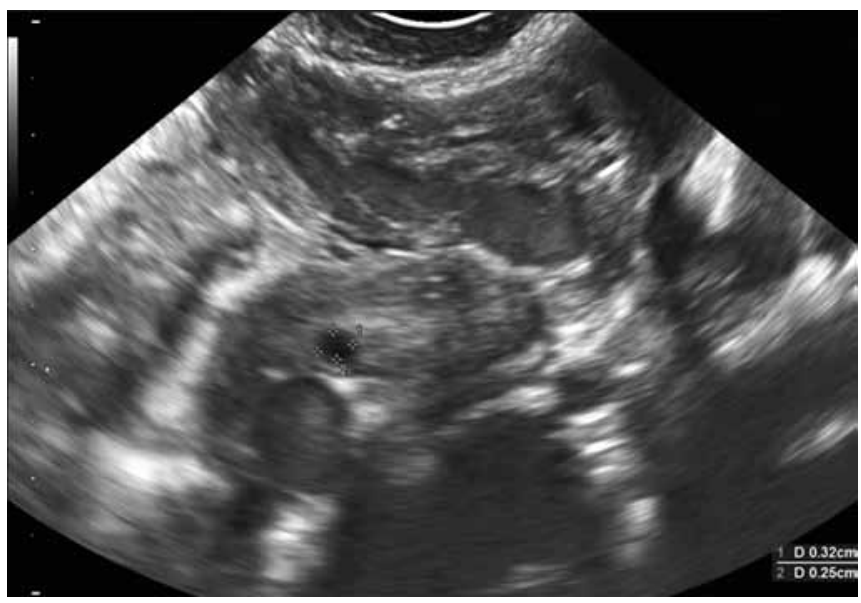

Fig. 4B: Transvaginal scan of the ovary. Note diminished ovarian volume and absence of growing follicles

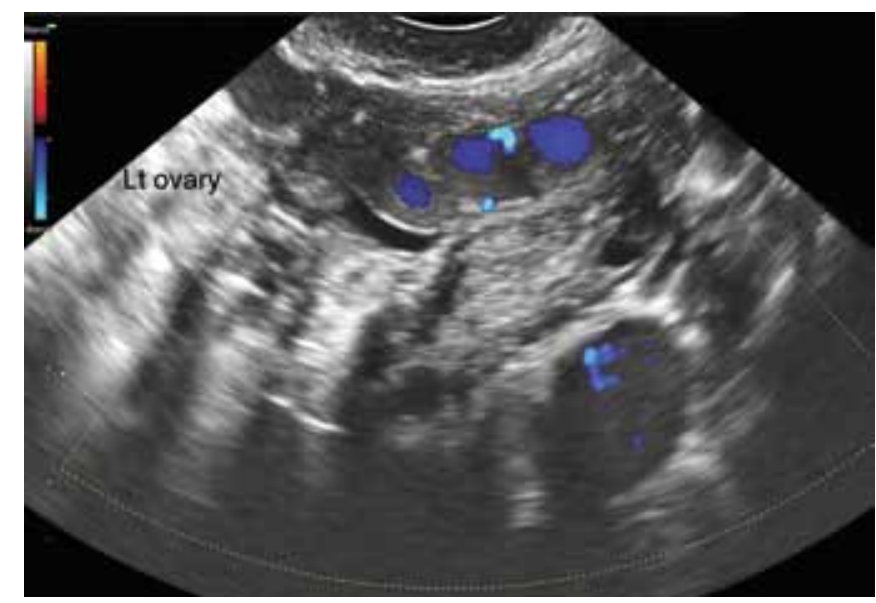

Fig. 4C: Color Doppler image of the same ovary. Note absence of intraovarian blood flow 


\section{Case 5}

A 35-year-old nulliparous patient presents for the assessment of primary infertility. She has suffered from chronic pelvic pain for 4 years. Because of worsening menstrual pain she was admitted twice to emergency care facility. Her past medical history is nonsignificant. She is taking ibuprofen as needed. There is no family history of cancer, gynecological problems, no history of birth defects and infertility. General physical examination reveals well-developed and wellnourished reproductive age patient in no acute distress. Her abdomen is soft, nontender, with no masses and/or hepatosplenomegaly. Her Pap smear was performed a year ago and was normal. Neisseria gonorrhoeae/Chlamydia trachomatis cultures were negative. Semen analysis of her husband was normal.

Pelvic examination reveals diffusely enlarged uterus and bilateral adnexal masses. You decide to order pelvic ultrasound (findings shown on Figs 5A to D).

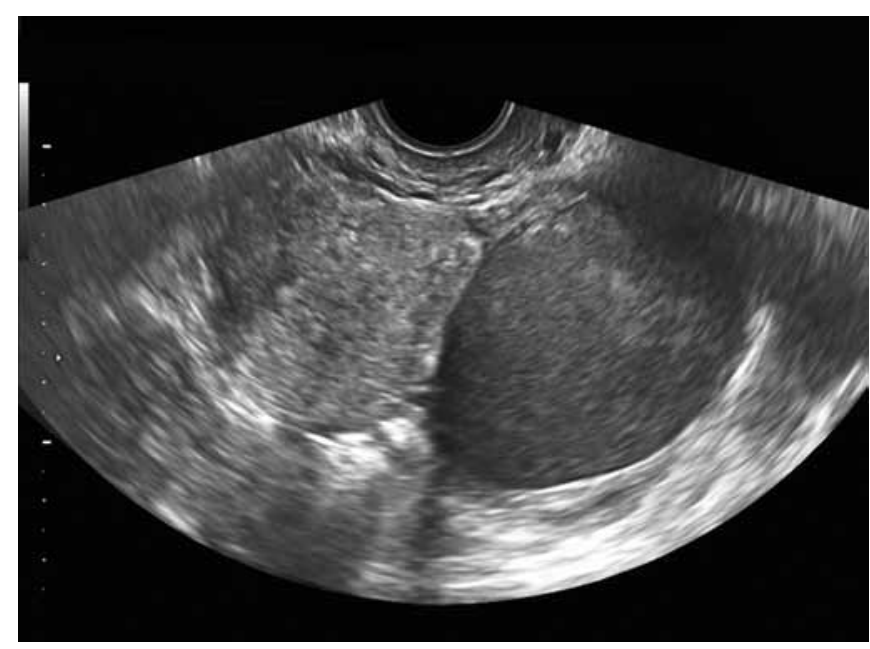

Fig. 5A: Transvaginal ultrasound of a complex ovarian lesion. Note homogenous 'ground glass' appearance

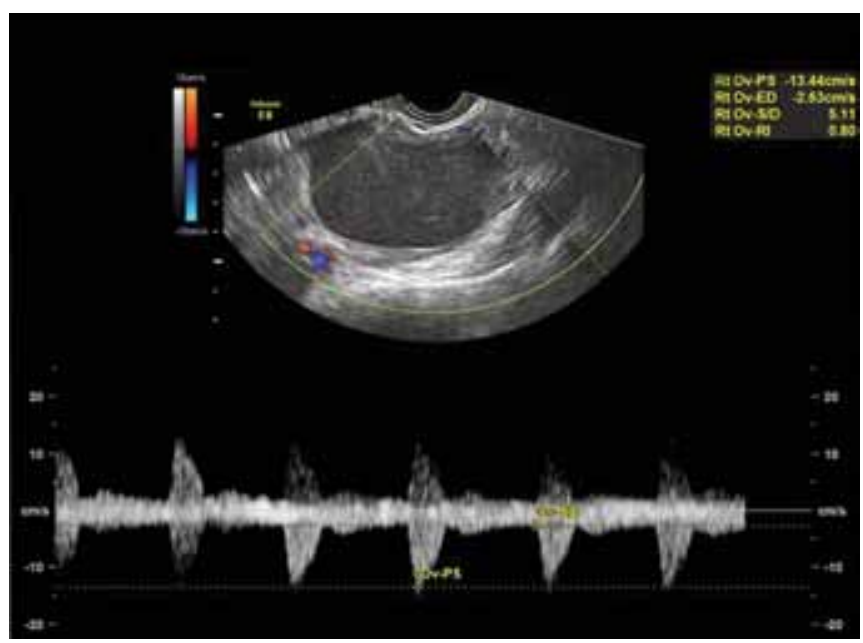

Fig. 5C: Pulsed Doppler waveform analysis shows moderate to high vascular impedance (RI 0.80)

\section{Case 6}

A 29-year-old primary infertile patient presents to your office for infertility assessment. Her history is significant for STD and two episodes of PID. Age of first intercourse was 14 years, onset of menarche at age 13. Her last menstrual period was seven days ago and was normal. She complains of chronic pelvic pain. Her past medical history (other than PID) is nonsignificant. Her vital signs are within normal limits. Her physical examination is significant for bilateral adnexal fullness. Figures $6 \mathrm{~A}$ and $\mathrm{B}$ demonstrate her pelvic ultrasound findings.

\section{Case 7}

A 32-year-old (G3P2012) woman presents to your office because of secondary amenorrhea. The patient's menstrual cycles had previously been normal (28/5). Her history is significant for an incomplete abortion 6 months ago. Five months ago she underwent a dilatation and curettage (D\&C)

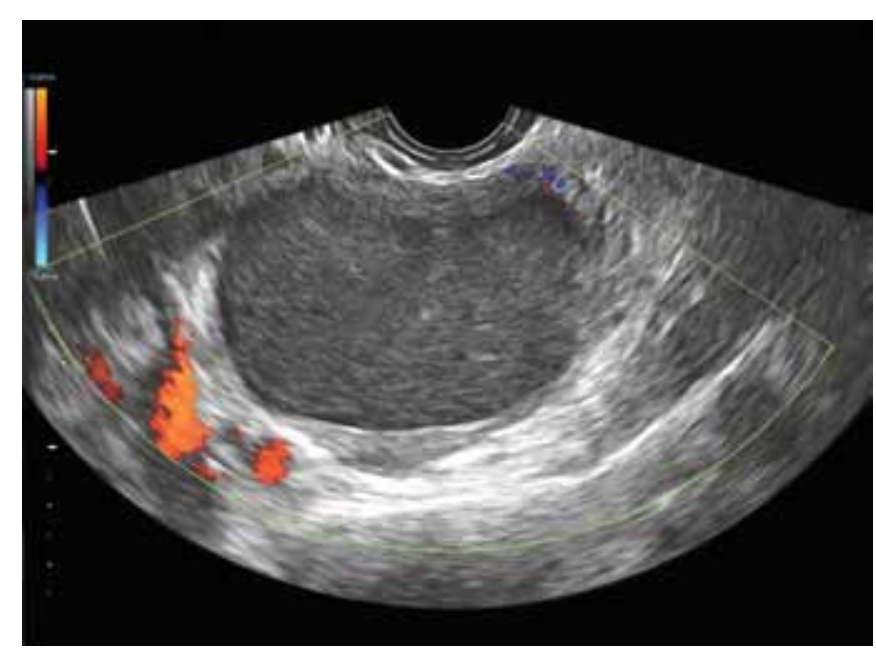

Fig. 5B: Color Doppler ultrasound of the same patient. Note discrete blood flow signals depicted at the periphery of the complex cystic lesion

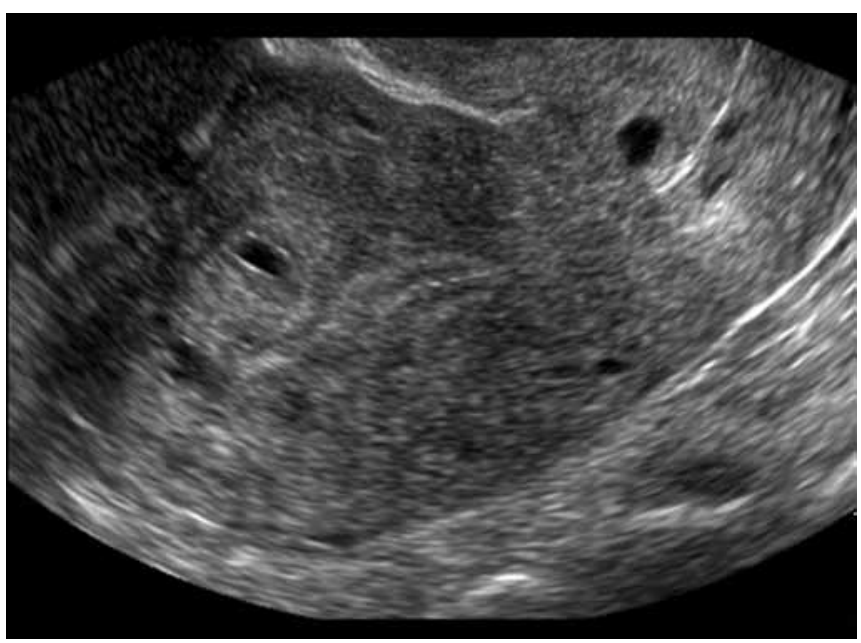

Fig. 5D: Transvaginal ultrasound of a 'Swiss cheese' appearance of the myometrium 


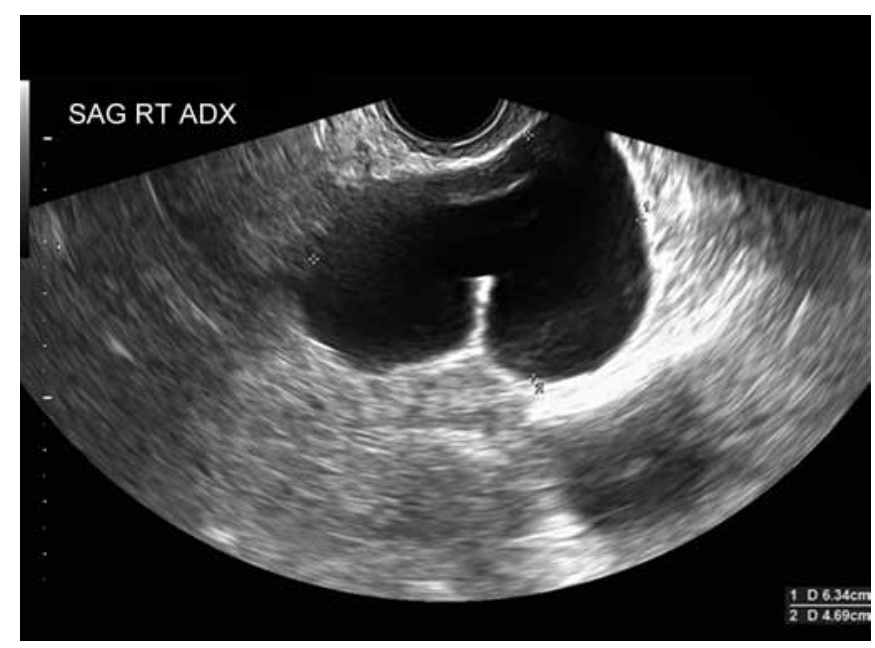

Fig. 6A: Complex adnexal mass with incomplete septation

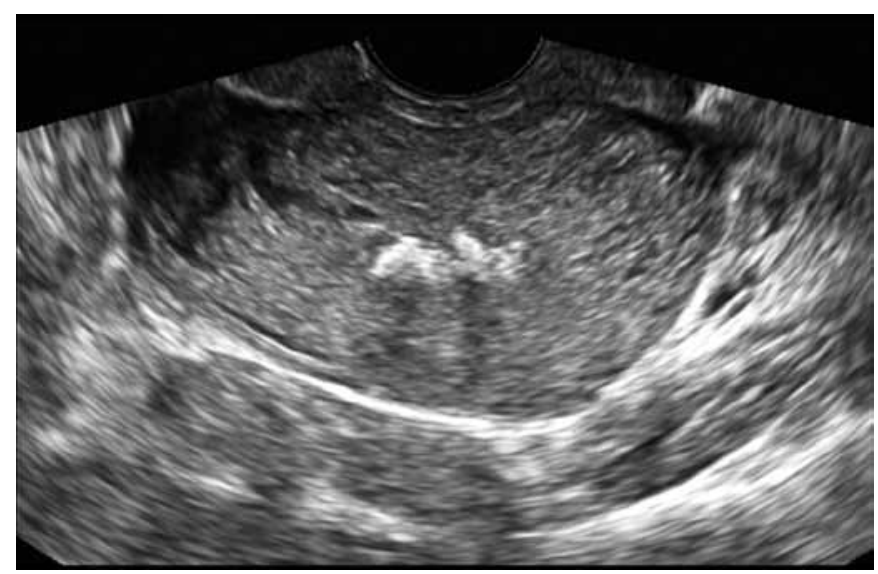

Fig. 7: Transvaginal ultrasound. Note hyperechogenic bands within the central part of the uterus. The endometrium could not be delineated

for heavy bleeding associated with presence of residual products of conception. Her menarche was at 13 years. General physical and pelvic examinations were normal. There are no symptoms suggestive of ovarian failure (patient does not complain of hot flashes, insomnia, and similar menopausal symptoms). There is no evidence of galactorrhea.

\section{Case 8}

A 32-year-old female (G3P0030) presents to your office with history of recurrent pregnancy loss at 8,9 and 12 weeks of gestation. She has had 3 miscarriages within the past 4 years. She is in a monogamous relationship with her husband and she wants to have a child. Her menstrual cycles are regular (28/6 days). Her history is significant for dysmenorrhea. Onset of menarche was at 12 years, age of first intercourse was 17 years, with no history of sexually transmitted diseases. She is currently not on any medications.

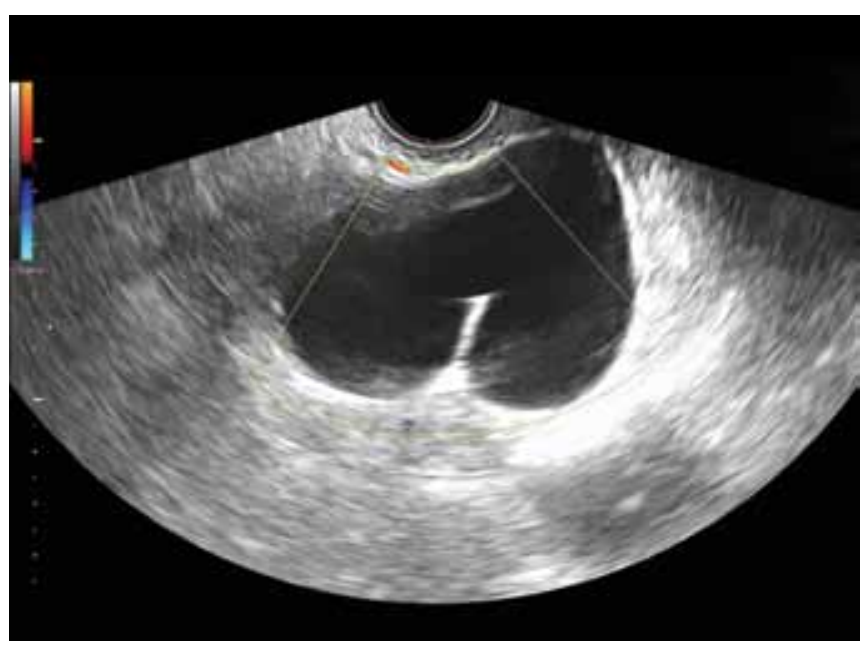

Fig. 6B: Color Doppler of the same adnexal mass. Note absence of blood flow signals within the incomplete septation

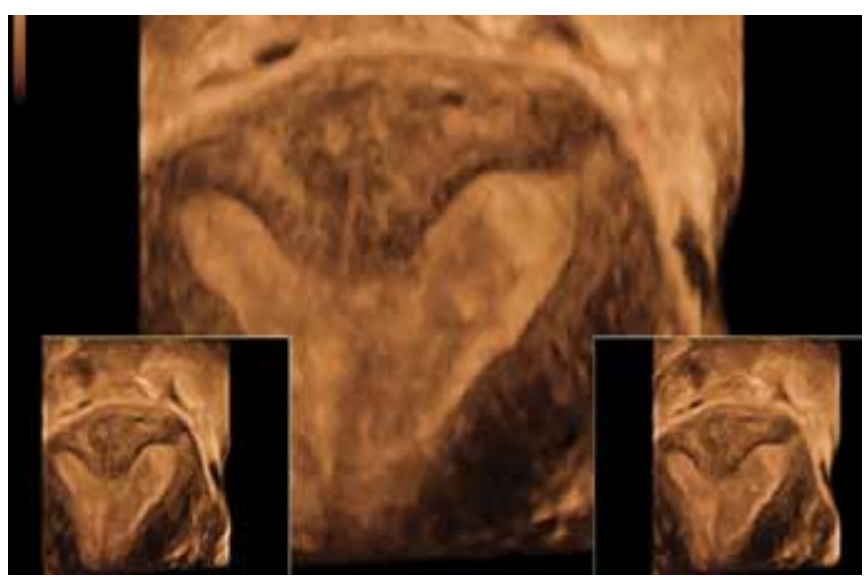

Fig. 8: Three-dimensional ultrasound of the uterus. Note complete division of the uterine cavity and convex shape of the uterine fundus

Her vital signs and her physical examination were normal. Figure 8 illustrates her 3D ultrasound findings.

\section{Case 9}

A 38-year-old G2P1011 female complains of menorrhagia for the past 1.5 years. Her periods occur every 28 days and used to last for 5 days, but are now lasting up to 10 to 14 days at a time. One year ago she was diagnosed with spontaneous abortion at 8 weeks. On physical examination, she is visibly pale. Her vital signs are within normal limits. Her last menstrual period was 3 weeks ago. On pelvic examination, the uterus is enlarged, and no adnexal masses are palpated. Laboratory studies revealed negative beta-hCG. Her hemoglobin level is $9.0 \mathrm{gm} / \mathrm{dl}$ and her hematocrit is $22 \%$. She is married for 8 years and wants to get pregnant.

Transvaginal ultrasound showed irregular contours of the uterine cavity and an order was placed for saline infusion sonography (SIS). Saline infusion sonography findings are demonstrated in Figures 9A and B. 


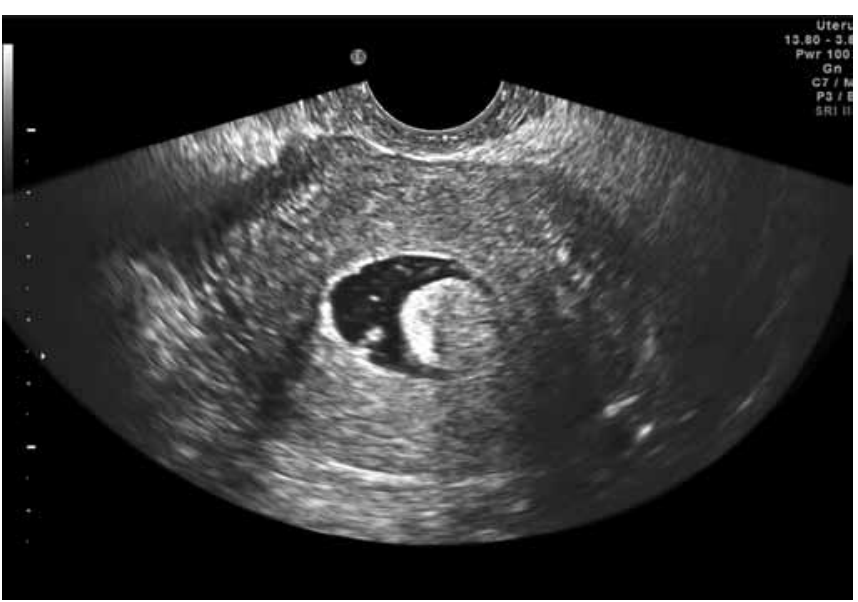

Fig. 9A: Saline infusion sonography of case 9. Note regular round lesion protruding into the uterine cavity

\section{Case 10}

A 29-year-old primary infertile patient is getting prepared for her IVF/ET cycle. Recently she noticed that her periods are prolonged with evidence of 3 to 4 days spotting. Her past medical history is nonsignificant. Her general physical and pelvic examinations were within the normal limits. Her Pap smear and Gonorrhea and Chlamydia cultures were normal. Her husband was diagnosed with oligoasthenozoospermia. Pelvic ultrasound was performed and revealed thickened and hyperechogenic endometrium in early postmenstrual phase. An order was placed for SIS and results are shown on Figure 10.

\section{DISCUSSION}

\section{Case 1: Polycystic Ovary Syndrome}

Polycystic ovary syndrome (PCOS) is diagnosed based on two out of the three Rotterdam criteria: oligomenorrhea and/ or anovulation, hyperandrogenism, and polycystic ovaries on ultrasound. ${ }^{1}$ Our patient presents with oligomenorrhea (irregular menstrual periods) and hyperandrogenism (acne and male pattern hair distribution). We must rule out pregnancy in any woman of reproductive age that is having menstrual irregularities (via beta-hCG). Serum follicle stimulating hormone (FSH) should be measured to rule out ovarian insufficiency. In patients with PCOS, FSH will be normal but luteinizing hormone (LH) will be elevated with an LH : FSH ratio usually greater than 2.

Transvaginal ultrasound is advantageous to transabdominal because it avoids the need for a full bladder and eliminates the difficulty of visualizing structures in the obese patient. Pelvic ultrasound in this patient demonstrated enlarged ovaries and an increased ovarian volume of approximately $15 \mathrm{cc}$ bilaterally (see Figs $1 \mathrm{~A}$ and B). Criteria for diagnosing polycystic ovaries on ultrasound is an ovarian

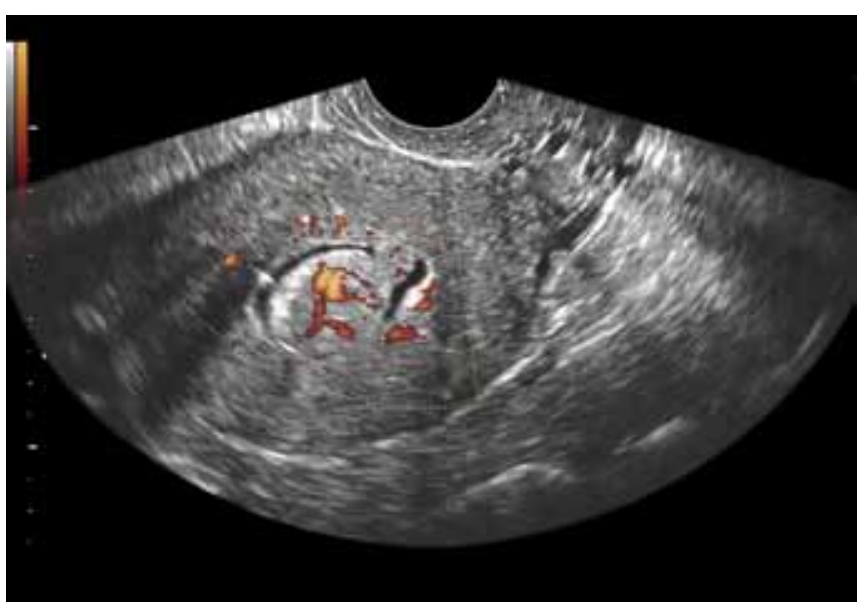

Fig. 9B: Power Doppler ultrasound of the same patient. Color Doppler displays vessels within the intracavitary lesion

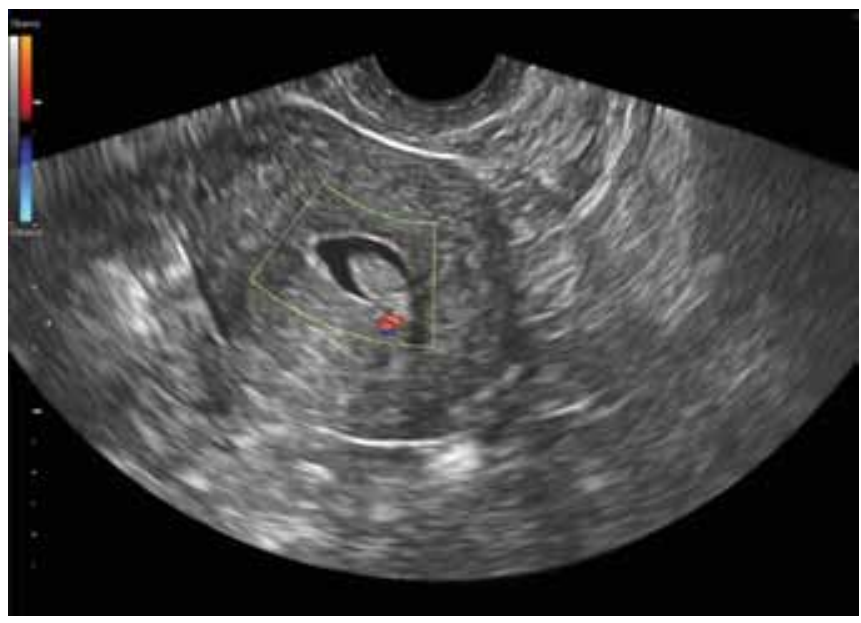

Fig. 10: Color Doppler image following SIS demonstrates hyperechogenic focal endometrial thickening. Note small feeding vessel

volume $>10 \mathrm{cc}$ or more than 10 follicles measuring between 2 and $9 \mathrm{~mm}^{1}$

Color Doppler studies in Figure 1C show changes in the ovarian vasculature which are associated with tonic hypersecretion of LH during the follicular phase of the menstrual cycle. There are higher uterine pulsatility index (PI) values; $\mathrm{PI}$ is a measure of the variability of blood velocity in a vessel. The higher PI values are possibly due to direct androgen vasoconstriction in arterial vessel walls, thereby reducing uterine perfusion. This has been cited as a possible cause of failed blastocyst implantation, increasing the incidence of miscarriages in PCOS patients. ${ }^{2}$ The ovaries are usually enlarged in PCOS patients; however, they can also be of normal size. Visualization of the stromal area can provide a diagnostic clue to differentiate PCOS from the multifollicular ovary; it is hypertrophic in PCOS whereas it is not increased in size in multifollicular ovaries. ${ }^{3}$ The presence of multiple ovarian cysts resembling PCOS on ultrasound have been found in $92 \%$ of women with idiopathic hirsutism, $87 \%$ with oligomenorrhea and $82 \%$ in both premenopausal type 2 
diabetics and congenital adrenal hyperplasia. ${ }^{4-6}$ Therefore, it is important to rule out these disorders in a woman presenting with symptoms of PCOS.

Other diseases that can cause anovulation, oligomenorrhea and hyperandrogenism must be excluded before diagnosing PCOS. The differential diagnosis of oligomenorrhea can be narrowed by ordering TSH to rule out thyroid dysfunction and prolactin to rule out hyperprolactinemia. All women with possible PCOS should be screened for nonclassical congenital adrenal hyperplasia (NCCAH) with 17-hydroxyprogesterone levels. ${ }^{7}$ Androgen-secreting ovarian/adrenal tumors and ovarian hyperthecosis can mimic PCOS with hirsutism and acne. However, pertinent clues from the history, such as age, timing and severity of symptoms and presence of virilization help distinguish the two. Women with PCOS are classically premenopausal and have a history of irregular periods since menarche whereas women with androgen-secreting tumors are usually postmenopausal and present with recent, sudden onset of hirsutism and virilization (frontal balding, severe acne, clitoromegaly, increased muscle mass or voice deepening). ${ }^{8}$

Women with PCOS are at increased risk for diabetes, cardiovascular disease, infertility, endometrial cancer, mood disorders and sleep apnea. Obesity and insulin resistance are highly prevalent among women with PCOS, therefore they are advised to control weight with diet and exercise. In addition, blood pressure, body mass index (BMI), fasting lipid panel and a 2 hour oral glucose tolerance test (OGTT) should be obtained at the initial visit. ${ }^{9}$ Patients with a normal glucose tolerance test should be rescreened at least once every two years and those with an abnormal test should be screened annually to monitor development of type 2 diabetes.

Women with PCOS are frequently unable to conceive due to their anovulatory menstrual cycle; serum progesterone can be measured to assess whether or not ovulation has occurred. If not, clomiphene citrate can be used to induce ovulation. Ultrasound can be used to monitor the status of ovulation. Color Doppler in Figure 1C demonstrates prominent intraovarian vascular network, which is considered highrisk for ovulation induction as there is an increased risk of ovarian hyperstimulation syndrome (OHSS). Ovarian hyperstimulation syndrome is a potentially life-threatening complication that presents with massive ovarian enlargement and third-spacing of intravascular fluid leading to renal failure, hypovolemic shock, thromboembolism, acute respiratory distress syndrome, and death. ${ }^{10,11}$ The proposed mechanism is a possible increased delivery of gonadotropins due to increased blood flow, leading to an excessive ovarian response. Evaluation of the vasculature via 3D power Doppler may be helpful to learn more about OHSS. ${ }^{12}$
The unopposed estrogen due to anovulatory cycle places women with PCOS at increased risk of endometrial cancer. Routine use of transvaginal ultrasound to screen for cancer is not recommended for premenopausal women. ${ }^{13}$ Endometrial hyperplasia is prevented with a progestin-containing oral contraceptive which also alleviates acne and regulates the menstrual cycle. ${ }^{14}$

Finally, women with PCOS should be screened for depression, anxiety and sleep apnea. ${ }^{15}$ First-line treatment is weight reduction via diet and exercise as a lower BMI is associated with an improved likelihood of ovulation and conception and an overall improved quality of life. Polycystic ovary syndrome is progressive; therefore, the use of ultrasound with color Doppler can play a significant role in treatment and follow-up.

\section{Case 2: Luteinized Unruptured Follicle}

Luteinized unruptured follicle (LUF) can occur when there are presumptive signs of ovulation without release of an oocyte. The biphasic basal body temperature and normal serum progesterone level exclude anovulatory cycle as the cause of our patient's irregular bleeding. Ultrasound can also be used to confirm ovulation by measurement of mean follicular diameter, detection of follicular collapse, visualization of the corpus luteum and extraovarian signs (thickened endometrium and free fluid in the culde-sac). The estimated frequency of LUF syndrome is between 6 and 47\%. ${ }^{12}$ A precise etiology has yet to be established, but numerous theories have been proposed; these include impairment of the mid-cycle LH surge, absence of preovulatory progesterone rise, abnormalities of prostaglandin synthesis and an intrinsic oocyte abnormality. Micu et al conducted a prospective study which showed that women receiving nonsteroidal anti-inflammatory drugs (NSAIDs) for rheumatic disease had a greater incidence of the LUF syndrome as compared to nonexposed women (35.6 vs 3.4\%). ${ }^{16}$ Etoricoxib, a COX-2 inhibitor, was found to have the most potent effect ( $75 \%$ of all LUF cases) while diclofenac was less significant (15\%). Interestingly, there was a much higher frequency of LUF syndrome in patients with inactive rheumatic disease compared with active disease. ${ }^{16}$ There is an association between LUF syndrome and unexplained infertility, chronic pelvic infections and endometriosis.

Affected patients are advised to undergo follicular assessment via transvaginal ultrasound. The resistive index (RI) is a measure of blood flow impedance and exhibits normal variations throughout the menstrual cycle. In normal patients, preovulatory RI values are moderate to high $(0.56 \pm 0.06)$ and drop after the LH surge $(0.44 \pm 0.04)$. 
However, multiple studies have shown that RI values in LUF syndrome patients remain high throughout both follicular and luteal phases. In addition, endometrial dating is normal in these patients. ${ }^{17,18}$ Perifollicular blood flow around the time of ovulation seems to be regulated by $\mathrm{LH}$ and the decrease in blood flow velocity due to a persistently high vascular impedance is diagnostic of LUF syndrome. ${ }^{19}$

Two types of LUF syndrome have been identified: mature follicle LUF and premature luteinization LUF. In the first type, the release of an oocyte was not demonstrated following follicle maturation (serum estradiol reached 200 $\mathrm{pg} / \mathrm{ml}$ while serum progesterone remained less than 2.5 $\mathrm{ng} / \mathrm{ml}$ ). In the second type the serum progesterone increased above $2.5 \mathrm{ng} / \mathrm{ml}$ before follicular maturation was attained. Human chorionic gonadotropin (hCG) can be used alone or in combination with human menopausal gonadotropin (hMG) to correct mature follicle LUF, while gonadotropins can be used to speed up the maturation process in premature luteinization $\mathrm{LUF}^{20}$ If gonadotropin therapy is ineffective, the patient's next option is in vitro fertilization and embryo transfer (IVF-ET).

\section{Case 3: Luteal Phase Defect}

A patient who presents with shortened menstrual cycles and first-trimester pregnancy loss should be evaluated for luteal phase defect (LPD). Initial diagnostic studies should include a serum progesterone level (normal $=4.4$ to 28.0 $\mathrm{ng} / \mathrm{ml}$ ) and pelvic ultrasound during the mid-luteal phase (day 20-21 of menstrual cycle). Once a thickened endometrium is identified on ultrasound, endometrial biopsy may be performed to confirm dating. Luteal phase defect (also known as short luteal phase, luteal insufficiency, inadequate luteal phase and luteal phase deficiency) is characterized by lack of progesterone, a luteal phase of the menstrual cycle shorter than 11 days and an out-of-phase endometrium by 2 or more days. The exact etiology is unclear; however the cause may be an intrinsic defect in the formation of the corpus luteum or external factors such as strenuous exercise or ovulation stimulating drugs. ${ }^{12}$

The corpus luteum plays a significant role in the woman's reproductive cycle, especially in early pregnancy. Once ovulation has occurred, blood vessels from the theca layer invade the ruptured follicle beginning the formation of the corpus luteum. Luteal cells produce more LH receptors and through a positive feedback mechanism, amplify the production of progesterone. This amplification continues until the mid-luteal phase, which is characterized by peak values of blood $\mathrm{LH}$, progesterone and a low resistance index (RI) in corpus luteum blood vessels. As was mentioned previously, women with regular ovulatory cycles have variation in their ovarian RI values depending on the phase of menstrual cycle. During the follicular phase, a moderate to high RI (mean $0.56 \pm 0.06$ ) can be obtained at the rim of the follicle with a significant decline on the day of the LH peak $(0.44 \pm 0.04)$ with the nadir occurring at midluteal phase $(0.42 \pm 0.06)$. In women with LPD, there is no change in the intraovarian RI during the transition from follicular (RI 0.56 \pm 0.06 ) to luteal (RI $0.56 \pm 0.04$ ) phases, and also shows no changes between early, middle, and late luteal phases. ${ }^{12,21}$

Luteal phase defect constitutes one of the possible endocrine factors involved in recurrent pregnancy loss (RPL). There are a number of contributors that could potentially cause miscarriage such as impaired corpus luteum formation and decreased endometrial vascularity. A study by Glock et al demonstrated that decreasing corpus luteum volume before 8 weeks gestation was associated with increased risk of pregnancy loss. They used color and pulsed Doppler to determine the dominant ovary (one containing corpus luteum). The dominant ovary showed low impedance to blood flow with RI values between 0.39 and 0.49 while the contralateral nondominant ovary had RI values between 0.69 to 1.0. Patients with ovarian RI values of 0.74 from a dominant ovary and 0.79 in nondominant ovary experienced pregnancy loss. ${ }^{22}$ Glock and Brumsted also correlated intraovarian blood flow with values of progesterone throughout the menstrual cycle. Mean progesterone levels were significantly lower for patients with LPD as compared to controls. $^{23}$

A decline in endometrial vascularity is also associated with miscarriage as shown in a study by Chen et al. ${ }^{24}$ They compared the endometrial and subendometrial vascularity of healthy, fertile women and those with recurrent miscarriages. Using 3D ultrasound and power Doppler angiography, they found that the endometrial and subendometrial vascularization index, flow index and vascularization flow index were greatly reduced in the group with unexplained recurrent miscarriage. ${ }^{24}$

Luteal phase defect is a commonly encountered problem when using assisted reproductive technology (ART). After ovulation induction, supporting the luteal phase with progesterone or hCG has shown to improve pregnancy outcomes. ${ }^{25}$ Some authors found vaginal progesterone to be the most efficacious with the least side effects while hCG was associated with the risk of ovarian hyperstimulation. ${ }^{26}$ In some instances, the LPD associated with controlled ovarian hyperstimulation $(\mathrm{COH})$ for IVF was not correctable. For these cases they recommended freezing of all embryos and deferring transfer to an artificial estrogen progesterone or natural cycle. ${ }^{26}$

\section{Case 4: Premature Ovarian Failure}

Premature ovarian failure (POF), also known as primary ovarian insufficiency, is the cessation of menses for at 
least 4 months before the age of 40 years. ${ }^{27}$ Spontaneous POF occurs at a rate of 1 in 10,000 by age 20, 1 in 1,000 by age 30 , and 1 in 100 by age $40 .{ }^{28}$ Patients may present with menopause-like symptoms such as oligomenorrhea/ amenorrhea, vaginal dryness, hot flashes and dyspareunia which are characteristic of an estrogen deficiency state. Any woman $<40$ years old presenting with $>3$ consecutive months of menstrual irregularity warrants an evaluation for POF. ${ }^{29}$ Other causes of menstrual irregularity such as pregnancy and hyperprolactinemia should be ruled out. Definitive diagnosis is made via measurement of serum estradiol and serum FSH on day 3 of the menstrual cycle. An estradiol level of $<30 \mathrm{pg} / \mathrm{ml}$ and FSH $>30-40 \mathrm{IU} / 1$ in the presence of clinical symptoms is highly suggestive of POF. However, these values can be closer to normal if the patient is experiencing oligomenorrhea. Transvaginal ultrasound can assist in diagnosis by demonstrating a thin and hyperechogenic endometrium as seen in post-menopausal women (see Fig. 4A). The cut-off value for endometrial thickness is $5 \mathrm{~mm}$ in patients with menopause. There is an inverse relationship between uterine/ovarian size and the time since menopause; the uterus and ovaries will significantly decrease in size as the duration of menopause increases. ${ }^{30}$ Figure $4 \mathrm{~B}$ demonstrates a diminished ovarian volume and absence of growing follicles, while Figure $4 \mathrm{C}$ exhibits decreased intraovarian blood flow.

After diagnosing POF, transvaginal ultrasound can also be used to assess follicle count and function. Hubayter et al. injected a single dose of $300 \mathrm{IU} \mathrm{hrFSH}$ to 95 women with POF and 42 regularly menstruating controls. Antral follicles $>3 \mathrm{~mm}$ were detected in $73 \%$ of patients on pelvic ultrasound and both serum estradiol and progesterone levels correlated with follicular diameter. Estradiol concentration did not significantly increase in patients with POF as compared to controls. However, the evidence of progesterone secretion by larger follicles $(>8 \mathrm{~mm}$ ) suggests that luteinization is the main contributing factor to ovarian dysfunction in women with POF. ${ }^{31}$

Almost $90 \%$ of POF cases are idiopathic. ${ }^{27}$ Known causes can be classified as genetic, autoimmune, or environmental. Two common genetic causes are Turner syndrome and Fragile X syndrome premutation. Turner syndrome is characterized by 'streak' ovaries containing connective tissue and atretic follicles. ${ }^{32}$ If a patient presents with absent pubertal development, primary amenorrhea, and morphological characteristics of Turner syndrome (short stature, shield chest), karyotype is warranted. Aside from the monosomy resulting in Turner syndrome, there are various X deletions, duplications, and translocations that cause POF. Xq13 to $\mathrm{Xq} 26$, excluding $\mathrm{Xq} 22$, have been implicated as the region responsible for ovarian development and function. ${ }^{33}$ The phenotype of Fragile X syndrome depends on the number of trinucleotide repeats in the FMR1 gene. Alleles with $<40$ CGG repeats are normal, 55 to 200 are 'premutations' and $>200$ are full mutations. The full mutation involves methylation of the gene and no subsequent protein product; however the premutation has decreased protein but elevated levels of messenger RNA (mRNA). Since the premutation variant is the only one associated with POF, it is possible that the excess mRNA acts as a toxin. ${ }^{34-36}$

Other genetic causes implicated in POF are galactosemia and autosomal dominant premature ovarian failure. The hypogonadism of galactosemia is thought to be due to the toxic effects of galactose metabolites. Patients who are heterozygous for the mutation do not experience $\mathrm{POF}^{37,38} \mathrm{~A}$ study by Kasippillai et al identified a heterozygous nonsense mutation with autosomal dominant inheritance when they evaluated a family with 9 women who developed menopause at age 30 in consecutive generations. ${ }^{39}$

Premature ovarian failure has an autoimmune basis in a specific subset of patients. At the time of diagnosis, women should undergo testing for serum antiadrenal and anti-21 hydroxylase antibodies as an estimated $3 \%$ will have concurrent autoimmune adrenal insufficiency. ${ }^{40}$ Autoimmune oophoritis may be part of a polyglandular autoimmune syndrome, so it is imperative to screen for other conditions, especially adrenal insufficiency and hypothyroidism. ${ }^{41}$ Serum antiovarian antibody testing has poor predictive value and ovarian biopsy is not recommended. ${ }^{42,43}$

Chemotherapy, radiation and surgery are implicated as environmental causes of POF. Both primordial and maturing follicles are decreased in the ovaries of women receiving chemotherapy ${ }^{44,45}$ Patients frequently develop amenorrhea and menstruate upon cessation of treatment. ${ }^{46,47}$ In contrast to chemotherapy, radiation is directly toxic to oocytes and is age and dose-dependent. ${ }^{48}$ Another iatrogenic cause of POF is surgery; aside from oophorectomy, hysterectomy is also associated with earlier menopause possibly due to damage of ovarian blood supply. ${ }^{49}$

Treatment goals in patients with POF are alleviation of physical and emotional discomfort and prevention of complications. Estrogen-progestin hormone replacement therapy (HRT) is recommended to prevent osteoporosis and offer relief from vasomotor symptoms and vaginal dryness. ${ }^{50,51}$ Premenopausal HRT should continue until the natural age of menopause at approximately age $50 .{ }^{52}$ Women with POF who desire fertility are candidates for IVF with oocyte donation; the cumulative chance of achieving pregnancy after 3 cycles is up to $90 \% .{ }^{53}$ Those with an autoimmune basis may benefit from immunosuppression as a method of ovulation induction; however, this treatment runs the risk of iatrogenic Cushing's syndrome. ${ }^{54}$ 
Intermittent resumption of ovarian function is not uncommon; some women continue to menstruate and even retain fertility. Bidet et al found that 86/358 (24\%) of women with POF exhibited signs of continued ovarian function and $15(4.4 \%)$ had spontaneous pregnancies. Out of the 21 spontaneous pregnancies, 16 were viable. Factors predictive of ovarian function were a positive family history of POF, secondary amenorrhea, presence of follicles at ultrasound, and inhibin B and estradiol levels. Nonpredictive factors included association with an autoimmune disease, antimullerian hormone level, the presence of follicles on biopsy and genetic abnormalities. ${ }^{55}$ As exhibited in this study, ultrasound is a superior tool not only for diagnosis, but also for predicting prognosis in women with POF.

\section{Case 5: Ovarian Endometrioma}

A premenopausal woman presenting with dysmenorrhea and bilateral adnexal masses raises strong suspicion for endometriosis. Endometrioma is a mass of ectopic endometrial tissue that grows in the ovary. The etiology is imprecise although the current hypothesis suggests a retrograde flow of menstrual blood with subsequent deposition on the ovaries. Ovarioscopy and microbiopsy have demonstrated that endometriomas are formed by invagination of the ovarian cortex which already contains active implants. ${ }^{56}$ A patient with endometriosis presents with chronic pelvic pain of various types depending on the location of ectopic tissue: dysmenorrhea (pain with menstruation), dyspareunia (pain with intercourse) and/or dyschezia (pain with defecation).

Ultrasound plays a vital role in the assessment of adnexal masses. Sonographic findings suggestive of endometrioma include a 'ground glass' appearance in a thick walled cyst (unilocular or multilocular) and homogenous low to medium level echoes (see Fig. 5A). Demographics become very useful as these findings apply to premenopausal women. On the other hand, a 'ground glass' appearance in a postmenopausal woman warrants evaluation for malignancy. ${ }^{57}$ Color Doppler allows for visualization of vascularity within an endometrioma (see Fig. 5B) and can be used to confirm diagnoses after initial evaluation. Our patient exhibits moderate to high vascular impedance as demonstrated in Figure 5C. Impairment in uterine artery blood flow has been shown to affect fertility in patients with endometriosis. Using transvaginal ultrasound with Color Doppler, Porpora et al found significant RI alterations $(>0.8)$ in women with ovarian endometriomas. At follow-up three months after laparoscopic surgery, there was improvement in uterine artery flow and subsequently higher pregnancy rates. ${ }^{58}$

CA 125 is a tumor marker that may be elevated in a wide array of gynecological and nongynecological conditions.
Though it is not specific for endometriosis, measuring its levels can help stage the severity. Concentrations $>100 \mathrm{IU} / \mathrm{ml}$ are associated with peritoneal disease, adhesions and/or ruptured endometrioma. ${ }^{59}$ Another finding indicative of severe disease is the presence of 'kissing ovaries' on ultrasound, i.e. ovaries so massive that they practically touch or 'kiss' ${ }^{60}$

Endometriosis can be associated with adenomyosis, which is characterized by the in-growing of endometrium into the myometrium. It can present with pelvic pain, heavy menstrual bleeding, infertility, or it can be asymptomatic. Patients with adenomyosis have a diffusely enlarged uterus with a heterogenous myometrium containing multiple small cysts (see Fig. 5D). Color Doppler may demonstrate increased vascularity. Transvaginal ultrasound detects this condition with a sensitivity and specificity of 80 and $50 \%$ respectively. ${ }^{61}$

Definitive diagnosis and treatment of ovarian endometrioma is laparoscopic removal via cystectomy. Specifically, excision of the cyst wall is associated with lower rates of the following: recurrence, dysmenorrhea, dyspareunia, nonmenstrual pelvic pain and need for further surgery. It is also associated with increased rates of spontaneous pregnancy. ${ }^{62}$ Following surgery, long-term use of combined oral contraceptives has also shown to prevent recurrence. ${ }^{63}$

For women who wish to undergo IVF, surgical excision is not always recommended due to concern of decreased ovarian function. Endometriomas are only removed if the patient is in pain, if there is a complex or solid appearance of the ovarian endometrioma suggestive of malignancy, or if oocyte retrieval is hindered. ${ }^{64}$ Unfortunately women with severe endometriosis (stage III/IV) continue to experience poor embryo implantation and pregnancy rates. ${ }^{65}$

\section{Case 6: Tubal Cause of Infertility}

Pelvic inflammatory disease (PID) is an infection of the female genital tract and can involve the uterus, fallopian tubes and/or ovaries. It is associated with sexually transmitted infections, especially Neisseria gonorrhoeae and Chlamydia trachomatis, but can be polymicrobial. Symptoms that should prompt clinical evaluation include a foul-smelling vaginal discharge, postcoital bleeding and urinary frequency, while fever and pelvic pain may be absent. ${ }^{66}$ It is imperative to diagnose and appropriately treat PID to prevent long-term complications such as chronic pelvic pain, ectopic pregnancy and infertility. Our patient's history of multiple STD's and PID in the setting of chronic pelvic pain raises suspicion for hydrosalpinx.

Hysterosalpingography (HSG) is an appropriate initial test to assess for tubal patency. Traditional HSG involves X-rays and it should only be done in the follicular phase. 
Ultrasonography can also be used to assess fallopian tubes without exposing the oocytes to radiation. B-mode, color Doppler and 3D hystero-contrast-salpingography (HyCo-Sy) are safe and efficacious methods for evaluation of fallopian tube patency without exposure to contrast dyes or radiation. ${ }^{12}$ Three-dimensional technique offers the possibility of simultaneous presentation of the uterine cavity and the corresponding tube.

Normal, healthy fallopian tubes are usually not visualized by ultrasound unless they contain fluid. 'Thick tubal walls' with fluid in the lumen are a sensitive and specific finding of acute PID. ${ }^{67}$ Postinflammatory scarring of the fallopian tubes can cause fusion of the fimbriae, impeding drainage of fluid into the peritoneum resulting in a condition called hydrosalpinx. Ultrasound will show fluid filled tubes with thin walls and incomplete septations (see Fig. 6A). There are discrete vascularization signals at the periphery of the fallopian tubes; however, there is no blood flow within the incomplete septation (see Fig. 6B).

Some women with hydrosalpinx are asymptomatic and may present only with infertility. Zhu et al proposed decreased nerve fibers in the isthmus causing decreased motility as the mechanism behind tubal factor infertility. ${ }^{68}$ Treatment options are surgery and/or IVF-ET. The presence of hydrosalpinx during IVF-ET has lower rates of pregnancy and implantation and a higher rate of pregnancy loss. There may be direct embryotoxicity, decreased endometrial receptivity or a mechanical mechanism where tubal fluid flushes out the embryo. ${ }^{69,70}$ Tubal occlusion via the sterilization procedure Essure may be an effective option to manage hydrosalpinx prior to IVF-ET. ${ }^{71}$ For surgical management, laparoscopy can help predict the prognosis of doing a bilateral salpingectomy $v s$ neosalpingostomy. Chanelles et al demonstrated that patients with at least one functioning tube have higher cumulative pregnancy rates than patients undergoing IVF after bilateral salpingectomy (63.3\% vs $50 \%$ respectively). ${ }^{72}$ They also showed that conservative neosalpingostomy had a spontaneous pregnancy rate of $30.04 \%$.

\section{Case 7: Intrauterine Adhesions (Asherman Syndrome)}

The majority of patients with intrauterine adhesions (synechiae) have some degree of trauma, resulting in fibrous scar tissue within the uterine cavity. Ninety percent of cases are related to curettage for missed or incomplete abortion, postpartum hemorrhage, or retained products of conception. ${ }^{73}$ Patients usually present with infertility as their chief complaint; however, they can also have amenorrhea, cyclic pelvic pain and/or recurrent miscarriages. Amenorrhea due to the presence of adhesions is termed Asherman syndrome.

Pregnancy should be ruled out in any woman of reproductive age presenting with secondary amenorrhea. If patient is not pregnant, the physician should conduct a progesterone withdrawal test to assess for ovulatory function. Bleeding following the progesterone challenge indicates anovulatory cycle. If progesterone withdrawal test is negative, estrogenprogesterone sequential test is indicated. If that is negative, serum FSH, LH and estradiol levels should be measured to detect the presence of ovarian function, which is intact in patients with Asherman syndrome. Transvaginal ultrasonography can have a very high sensitivity (80-90\%) with an experienced sonographer. ${ }^{74}$ Intrauterine adhesions are visualized as irregular echogenic 'cyst-like' areas in a normal size uterus. Furthermore, there is difficulty delineating the endometrium (Fig. 7). ${ }^{75}$ Other diagnostic methods include sonohysterogram and hysterosalpingogram (HSG) which are shown to have equal sensitivities. ${ }^{76}$ Hysteroscopy is the gold standard for diagnosis of intrauterine adhesions as it allows for concurrent treatment with minimal trauma to the surrounding endometrium. ${ }^{77}$

Ultrasonography is not the ideal diagnostic modality, however it can be useful in cases when hysteroscopy is difficult, e.g. cervical obstruction. Transabdominal ultrasound guidance during resection of synechiae has been shown to have lower rates of uterine perforation. It is also less expensive than laparoscopy-guided hysteroscopy. ${ }^{78}$ Furthermore, transvaginal ultrasound can help to predict prognosis postoperatively: women with a well-developed endometrial 'stripe' lining resumed menses after surgical resection, whereas women with a minimal lining did not resume menses. $^{79}$

\section{Case 8: Septate Uterus}

Septate uterus is a congenital anomaly in which there is a dividing septum within an otherwise normally shaped uterus, creating two endometrial cavities. It has a $0.5 \%$ prevalence and is classified as a Type $\mathrm{V}$ mullerian anomaly by the American Fertility Society classification system. ${ }^{80}$ The septate uterus originates from failure of midline septal regression after complete fusion of the mullerian ducts. It can range from a partial midline septation to complete longitudinal septation extending to the internal os. Compared to other congenital anomalies, it has the highest incidence of reproductive difficulties. Conception and implantation are not affected, as women with uterine anomalies have similar pregnancy rates as their normal counterparts. ${ }^{81}$ However, they have a heightened risk of bad outcomes; complications include spontaneous abortion (21\%-44\%) and preterm delivery 
(12\%-33\%). ${ }^{82}$ Arcuate uterus is a much less severe variant that has a small midline septum with minimal fundal cavity indentation. $^{83}$

X-ray hysterosalpingography allows excellent visualization of the uterine cavity outline; however disadvantages include timing (must be done in follicular phase), use of contrast, exposure to radiation, and lack of delineation of external uterine contour. ${ }^{84} \mathrm{MRI}$ is useful in detecting complex anomalies but it is not routinely used in gynecological evaluation due to the advent of other less costly approaches.

Two-dimensional transvaginal ultrasonography has relatively low accuracy but can be used as a screening tool. B-mode ultrasound depicts septate uterus as two endometrial echoes in the uterine cavity. Color Doppler can be used to determine the presence of vascularization in the septum, which has been correlated with pregnancy loss. ${ }^{12}$ Saline infusion sonohysterography (SIS) is another method of visualizing the intrauterine space and structures however, like X-ray HSG, it is invasive and does not illustrate the external fundal contour.

Three-dimensional transvaginal ultrasonography has a diagnostic accuracy equal to hysteroscopic laparoscopy in diagnosing septate uterus as it depicts the uterine cavity, myometrium and external uterine contour. ${ }^{85,86}$ By $3 \mathrm{D}$ ultrasound septate uterus is demonstrated as a partial or full midline division between the two uterine horns, which creates a concave shape of the uterine fundus (see Fig. 8). The septum length and rate of uterine distortion can help predict the risk of obstetrical complications. ${ }^{87}$ The diagnostic accuracy and low cost make 3D ultrasound an excellent screening and diagnostic tool for detection of uterine anomalies.

Hysteroscopy and laparoscopy allow for direct visualization of the intrauterine and abdominal cavity and are therefore considered the gold standard for diagnosis and treatment. Resection of the septum via hysteroscopy or hysteroscopic metroplasty have shown to improve pregnancy outcomes substantially. ${ }^{88}$

\section{Case 9: Intracavitary Leiomyoma (Fibroid)}

Leiomyomas are benign tumors of the uterus composed of smooth muscle and connective tissue; they can occur at various locations in the uterus: subserosal, intramural, submucosal and cervical. Approximately $25 \%$ of women with fibroids are symptomatic and present with varying degrees of pelvic pain and menorrhagia (heavy menstrual bleeding). ${ }^{89}$ Submucosal and/or intracavitary fibroids are the type most commonly implicated in infertility; their location causes a distortion of the endometrial cavity possibly leading to impaired implantation and/or uterine contractility. ${ }^{90}$ Our patient's heavy menstrual bleeding, previous miscarriage, difficulty conceiving and enlarged uterus are all pertinent of uterine cause of infertility.

Leiomyomas have varying appearance on ultrasound depending on their composition of smooth muscle and connective tissue. Compared to hysteroscopy as gold standard of diagnosis, saline infusion sonohysterography (SIS) has been shown to be a low-cost, less invasive and effective screening method. ${ }^{91}$ Figure $9 \mathrm{~A}$ illustrates the round lesion protruding from the endometrium into the uterine cavity. Addition of color Doppler shows multiple vessels within the fibroid (see Fig. 9B). The greater vascularity of this lesion allows it to be differentiated from an endometrial polyp, which typically has a solitary feeding vessel within a stalk. ${ }^{92}$ As leiomyomas enlarge, they may outgrow their blood supply leading to ischemia which is visible as calcific degeneration. This poorly vascularized environment is not adequate to support the fertilized ovum. ${ }^{12}$ Color Doppler will show higher vascular impedance in submucosal fibroids due to the presence of myometrial tissue. Furthermore, centrally located vessels in a fibroid have lower RI values than peripheral vessels and can be mistaken for malignancy. ${ }^{93}$

There are various proposed mechanisms that lead to subfertility and infertility in the patient with submucosal and intracavitary fibroids. These tumors express angiogenic factors such as basic fibroblast growth factor and platelet derived growth factor, which possibly lead to impaired implantation of the embryo. Furthermore, their mechanical presence may alter gamete transport via changes in uterine contractility. Inflammatory and fibrotic changes in the endometrium are also implicated in the increased risk of pregnancy loss. ${ }^{94}$

Treatment for submucosal and intracavitary fibroids is based on the presence of symptoms and plans for future childbearing. ${ }^{90}$ The traditional approach for fibroids was hysterectomy; however, hysteroscopic myomectomy is recommended for women who wish to conceive. ${ }^{95}$ For those undergoing IVF-ET, the presence of submucosal and intracavitary fibroids decreases chances of success. ${ }^{96}$ At the moment there is insufficient evidence to support that removal increases IVF-ET outcome.

\section{Case 10: Endometrial Polyp}

Endometrial polyps are hyperplastic masses that originate from the endometrium. They are a common cause of abnormal uterine bleeding in both premenopausal and postmenopausal women. Though most endometrial polyps are benign, some express oncogenic potential. ${ }^{97}$ They are also implicated in subfertility and infertility. Our patient noted an abnormal bleeding pattern which should prompt evaluation. 
Transvaginal ultrasound is the preferred initial diagnostic study for assessment of women with abnormal uterine bleeding. For further evaluation of morphology, saline infusion sonography (SIS) and/or diagnostic hysteroscopy are recommended. Saline infusion sonography is superior to the other diagnostic tools when a clinician needs to differentiate between focal and diffuse endometrial thickening. ${ }^{98}$ The presence of intracavitary fluid allows for better visualization of the intracavitary lesions, such as endometrial polyp. ${ }^{12}$ Saline infusion sonography will demonstrate hyperechogenic endometrial thickening in a focal area (see Fig. 10). Addition of color Doppler may show a solitary feeding vessel. Endometrial polyps may be difficult to differentiate from intracavitary fibroids; however a few sonographic characteristics can assist in making a final diagnosis. The presence of a single vessel, an acute angle, homogeneous echogenicity and intact endometrialmyometrial interface in the lesion significantly increases the likelihood of endometrial polyp. ${ }^{99}$

Due to her husband's diagnosis of oligoasthenozoospermia, our patient must undergo IVF-ET to achieve a potentially successful pregnancy. However, her abnormal uterine bleeding and subsequent discovery of endometrial polyp on ultrasound can decrease chances of success. Similar to submucosal and intracavitary leiomyomas, polyps can cause recurrent failure of embryo transplantation. ${ }^{100}$ Hysteroscopic resection of endometrial polyps is effective in improving pregnancy rates $(63 \%$ vs $28 \%)$. Polypectomy is beneficial for both spontaneous pregnancies and those from assisted reproductive technology (ART) ${ }^{101}$ It is recommended for our patient to undergo removal of the endometrial polyp to increase chances of IVF-ET success.

\section{REFERENCES}

1. Rotterdam ESHRE/ASRM-Sponsored PCOS consensus workshop group. Revised 2003 consensus on diagnostic criteria and long-term health risks related to polycystic ovary syndrome (PCOS). Hum Reprod 2004;19(1):41.

2. Battaglia C, Artini PG, D'Ambrogio G, et al. The role of color Doppler imaging in the diagnosis of polycystic ovary syndrome Am J Obstet Gynecol 1995;172(1):108-113.

3. Robert Y, Dubrulle F, Gaillandre L, et al. Ultrasound assessment of ovarian stroma hypertrophy in hyperandrogenism and ovulation disorders: visual analysis versus computerized quantification. Fertil Steril 1995;64(2):307-312.

4. Adams J, Polson DW, Franks S. Prevalence of polycystic ovaries in women with anovulation and idiopathic hirsutism. Br Med J (Clin Res Ed) 1986;293(6543):355-359.

5. Conn JJ, Jacobs HS, Conway GS. The prevalence of polycystic ovaries in women with type 2 diabetes mellitus. Clin Endocrinol (Oxf) 2000;52(1):82-86.

6. Hague WM, Adams J, Rodda C, et al. The prevalence of polycystic ovaries in patients with congenital adrenal hyperplasia and their close relatives. Clin Endocrinol (Oxf) 1990;33(4): 501-510.

7. New MI. Nonclassic 21-hydroxylase deficiency. Fertil Steril 2006;86 Suppl 1:S2.

8. Derksen J, Nagesser SK, Meinders AE, et al. Identification of virilizing adrenal tumors in hirsute women. N Engl J Med 1994;331:968-973.

9. Salley KE, Wickham EP, Cheang KI, et al. Glucose intolerance in polycystic ovary syndrome: a position statement of the Androgen Excess Society. J Clin Endocrinol Metab 2007;92(12): 4546-4556.

10. Elchalal U, Schenker JG. The pathophysiology of ovarian hyperstimulation syndrome: views and ideas. Hum Reprod 1997;12(6):1129-1137.

11. Kaiser UB. The pathogenesis of the ovarian hyperstimulation syndrome. N Engl J Med 2003;349(8):729-732.

12. Kupesic S, Kurjak A. Color Doppler and three-dimensional ultrasound imaging in infertility. Color Doppler, 3D and 4D ultrasound in gynecology, infertility and obstetrics. 2nd ed. New Delhi: Jaypee Brothers, 2011, p. 115-130.

13. Dijkhuizen FP, Brölmann HA, Potters AE, et al. The accuracy of transvaginal ultrasonography in the diagnosis of endometrial abnormalities. Obstet Gynecol 1996;87(3):345-349.

14. Mueck AO, Seeger H, Rabe T. Hormonal contraception and risk of endometrial cancer: a systematic review. Endocr Relat Cancer 2010;17(4):R263-271.

15. Veltman-Verhulst SM, Boivin J, Eijkemans MJ, Fauser BJ. Emotional distress is a common risk in women with polycystic ovary syndrome: a systematic review and meta-analysis of 28 studies. Hum Reprod Update 2012;18(6):638-651.

16. Micu MC, MIcu R, Ostensen M. Luteinized unruptured follicle syndrome increased by inactive disease and selective cyclooxygenase 2 inhibitors in women with inflammatory arthropathies. Arthritis Care \& Research 2011;63(9):1334-1338.

17. Kupesic S, Kurjak A. The assessment of normal and abnormal luteal function by transvaginal color doppler sonography. Eur J Obstet Gynecol and Reprod Bio 1997;72(1):83-87.

18. Tamura H, Takasaki A, Taniguchi K, Matsuoka A, Shimamura K, Sugino N. Changes in blood-flow impedance of the human corpus luteum throughout the luteal phase and during early pregnancy. Fertil Steril 2008;90(6):2334-2339.

19. Zaidi J, Campbell S, Pittroff R. Ovarian stromal blood flow in women with polycystic ovaries: a possible new marker for diagnosis? Hum Reprod 1995;10(8):1992-1996.

20. Check JH, Dietterich C, Nowroozi K, Wu CH. Comparison of various therapies for the luteinized unruptured follicle syndrome. Int J Fertil 1992;37(1):33-40.

21. Kupesic S, Kurjak A, Vujisic S. Luteal phase defect: comparison between Doppler velocimetry, histological and hormonal markers. Ultrasound Obstet Gynaecol 1997;9(2):105-112.

22. Glock JL, Blackman JA, Bader GJ. Prognostic significance of morphologic changes of the corpus luteum by transvaginal ultrasound in early pregnancy monitoring. Obstet Gynecol 1995;85(1):37-41.

23. Glock JL, Brumsted JR. Color flow pulsed Doppler ultrasound in diagnosing luteal phase defect. Fertil Steril 1995;64:500-504.

24. Leining C, Song Q, Li H, Chen C, Xing F, Yu Y. A comparison of endometrial and subendometrial vascularity assessed by threedimensional ultrasonography and power doppler angiography between healthy fertile women and women with unexplained primary recurrent miscarriage. Fertil Steril 2011;95(3): $1127-1129$. 
25. Munoz E, Taboas E, Portela S, Aguilar L, Fernandez I, Munoz L, Bosch E. Treatment of luteal phase defects in assisted reproduction. Curr Drug Targets 2013;14(8):832-842.

26. Check JH. Luteal phase support for in vitro fertilization-embryotransfer-present and future methods to improve successful implantation. Clin Exp Obstet Gynecol 2012;39(4):422-428.

27. Nelson LM. Clinical practice. Primary ovarian insufficiency. N Engl J Med 2009;360(6):606-614.

28. Beck-Peccoz P, Persani L. Premature ovarian failure. Orphanet J Rare Dis 2006;1(9):1750-1772.

29. Mishell DRJ, Stenchever MA, Droegemueller W, Herbst AL. Primary and secondary amenorrhea. In: comprehensive gynecology, 3rd ed, Mosby, St. Louis 1997. p. 1043.

30. Kupesic S. Clinical sonographic pearls. In: Kupesic S, editor. Color Doppler, 3D and 4D ultrasound in gynecology, infertility and obstetrics. 2nd ed. New Delhi: Jaypee Brothers Medical Publishers Pvt Ltd. 2011, p. 270-272.

31. Hubayter ZR, Popat V, Vanderhoof VH. A prospective evaluation of antral follicle function in women with 46,XX spontaneous primary ovarian insufficiency. Fertil Steril 2010;94(5):1769-1774.

32. Albright F, Smith PH, Fraser R. A syndrome characterized by primary ovarian insufficiency and decreased stature: report of 11 cases with a digression on hormonal control of axillary and pubic hair. Am J Med Sci 1942;204:625-648.

33. Schlessinger D, Herrera L, Crisponi L. Genes and translocations involved in POF. Am J Med Genet 2002;111(3):328-333.

34. Bodega $\mathrm{B}$, Bione $\mathrm{S}$, Dalprà L. Influence of intermediate and uninterrupted FMR1 CGG expansions in premature ovarian failure manifestation. Hum Reprod 2006;21(4):952-957.

35. Bretherick KL, Fluker MR, Robinson WP. FMR1 repeat sizes in the gray zone and high end of the normal range are associated with premature ovarian failure. Hum Genet 2005;117(4):376-382.

36. Tassone F, Hagerman RJ, Taylor AK. Elevated levels of FMR1 mRNA in carrier males: a new mechanism of involvement in the fragile-X syndrome. Am J Hum Genet 2000;66(1):6-15.

37. Kaufman FR, Kogut MD, Donnell GN. Hypergonadotropic hypogonadism in female patients with galactosemia. N Engl J Med 1981;304(17):994-998.

38. KnauffEA, Richardus R, Eijkemans MJ. Heterozygosity for the classical galactosemia mutation does not affect ovarian reserve and menopausal age. Reprod Sci 2007;14(8):780-785

39. Kasippillai T, MacArthur DG, Kirby A. Mutations in eIF4ENIF1 are associated with primary ovarian insufficiency. J Clin Endocrinol Metab 2013;98(9):E1534-1539.

40. Bakalov VK, Vanderhoof VH, Bondy CA, Nelson LM. Adrenal antibodies detect asymptomatic auto-immune adrenal insufficiency in young women with spontaneous premature ovarian failure. Hum Reprod 2002;17(8):2096-2100.

41. Leshin M. Polyglandular autoimmune syndromes. Am J Med Sci 1985;290(2):77-88.

42. Novosad JA, Kalantaridou SN, Tong ZB, Nelson LM. Ovarian antibodies as detected by indirect immunofluorescence are unreliable in the diagnosis of autoimmune premature ovarian failure: a controlled evaluation. BMC Womens Health 2003; $3(1): 2$.

43. Khastgir G, Abdalla H, Studd JW. The case against ovarian biopsy for the diagnosis of premature menopause. $\mathrm{Br} \mathrm{J}$ Obstet Gynaecol 1994;101(2):96-98.

44. Nicosia SV, Matus-Ridley M, Meadows AT. Gonadal effects of cancer therapy in girls. Cancer 1985;55(10):2364-2372.

45. Siris ES, Leventhal BG, Vaitukaitis JL. Effects of childhood leukemia and chemotherapy on puberty and reproductive function in girls. N Engl J Med 1976;294(21):1143-1146.
46. Bakri YN, Pedersen P, Nassar M. Normal pregnancy after curative multiagent chemotherapy for choriocarcinoma with brain metastases. Acta Obstet Gynecol Scand 1991;70(7-8): 611-613.

47. Hershlag A, Schuster MW. Return of fertility after autologous stem cell transplantation. Fertil Steril 2002;77(2):419-421.

48. Baker TG. Radiosensitivity of mammalian oocytes with particular reference to the human female. Am J Obstet Gynecol 1971;110(5):746-761.

49. Farquhar CM, Sadler L, Harvey SA, Stewart AW. The association of hysterectomy and menopause: a prospective cohort study. $\mathrm{Br}$ J Obstet Gynaecol 2005;112:956-962.

50. Gallagher JC. Effect of early menopause on bone mineral density and fractures. Menopause 2007;14(3 pt 2):567-571.

51. Ostberg JE, Storry C, Donald AE. A dose-response study of hormone replacement in young hypogonadal women: effects on intima media thickness and metabolism. Clin Endocrinol (Oxf) 2007;66(4):557-564.

52. Kalantaridou SN, Nelson LM. Premature ovarian failure is not premature menopause. Ann N Y Acad Sci 2000;900:393-402.

53. Paulson RJ, Hatch IE, Lobo RA, Sauer MV. Cumulative conception and live birth rates after oocyte donation: implications regarding endometrial receptivity. Hum Reprod 1997; 12(4):835-839.

54. Kalantaridou SN, Braddock DT, Patronas NJ, Nelson LM. Treatment of autoimmune premature ovarian failure: case report. Human Reproduction 1999;14 (7):1777-1782.

55. Bidet M, Bachelot A, Bissauge E, Golmard JL, Gricourt S, Dulon J, Coussieu C, Badachi Y, Touraine P. Resumption of ovarian function and pregnancies in 358 patients with premature ovarian failure. J Clin Endocrinol Metab 2011;96(12):3864-3872.

56. Brosens IA, Puttemans PJ, Deprest J. The endoscopic localization of endometrial implants in the ovarian chocolate cyst. Fertil Steril 1994;61(6):1034-1038.

57. Busacca M, Riparini J, Somigliana E. Postsurgical ovarian failure after laparoscopic excision of bilateral endometriomas. Am J Obstet Gynecol 2006;195(2):421-425.

58. Porpora MG, Tomao F, Yazdanian D, Fuggetta E, Benedetti P, Piccioni MG, Benagiano G. Impaired uterine artery flow associated with the presence of ovarian endometrioma: preliminary results of a prospective study. J Ovarian Res 2014; 7(1): 18-21.

59. Cheng YM, Wang ST, Chou CY. Serum CA-125 in preoperative patients at high risk for endometriosis. Obstet Gynecol 2002; 99(3):375-380.

60. Ghezzi F, Raio L, Cromi A. 'Kissing ovaries': a sonographic sign of moderate to severe endometriosis. Fertil Steril 2005;83(1): 143-147.

61. Brosens J, Nandita M, Souza F, Barker G, Paraschos T, Winston RML. Endovaginal ultrasonography in the diagnosis of adenomyosis uteri: identifying the predictive characteristics. BJOG: Int J Obstet Gynaecol 1995;10(6):471-474.

62. Hart RJ, Hickey M, Maouris P. Excisional surgery versus ablative surgery for ovarian endometriomata. Cochrane Database Syst Rev 2008;16(2):CD004992. Available at: http://www.ncbi.nlm. nih.gov/pubmed/18425908.

63. Vercellini P, De Matteis S, Somigliana E. Long-term adjuvant therapy for the prevention of postoperative endometrioma recurrence: a systematic review and meta-analysis. Acta Obstet Gynecol Scand 2013;92(1):8-16.

64. Tsoumpou I, Kyrgiou M, Gelbaya TA, Nardo LG. The effect of surgical treatment for endometrioma on in vitro fertilization 
outcomes: a systematic review and meta-analysis. Fertil Steril 2009;92(1):75-87.

65. Harb HM, Gallos ID, Chu J, Harb M, Coomarasamy A. The effect of endometriosis on in vitro fertilisation outcome: a systematic review and meta-analysis. BJOG 2011;120(11): 1308-1320.

66. Soper DE. Pelvic inflammatory disease. Obstet Gynecol 2010;116(2 Pt 1):419-428.

67. Kupesic S, KurjakA, Pasalic L, Benic S. The value of transvaginal color Doppler in the assessment of pelvic inflammatory disease. J Ultrasound Med Biol 1995;6:733-738.

68. Zhu L, Chen J, Huang X, Xu H, Zhang X. Loss of nerve fibers in the oviduct isthmus in women with hydrosalpinx. Acta Histochem 2013;115(6):609-615.

69. Camus E, Poncelet C, Goffinet F. Pregnancy rates after invitro fertilization in cases of tubal infertility with and without hydrosalpinx: a meta-analysis of published comparative studies. Hum Reprod 1999;14(5):1243-1249.

70. Salpingectomy for hydrosalpinx prior to in vitro fertilization. Fertil Steril 2008;90(3):S67-68.

71. Arora P, Arora R, Cahill D. Essure ${ }^{\circledR}$ for management of hydrosalpinx prior to in vitro fertilisation: a systematic review and pooled analysis. BJOG 2014;PMID: 24393165. Available at: http://www.ncbi.nlm.nih.gov/pubmed/24393165

72. Chanelles O, Ducarme G, Sifer C, Hugues JN, Touboul C, Poncelet C. Hydrosalpinx and infertility: what about conservative surgical management? Eur J Obstet Gynecol Reprod Biol 2011;159(1):122-126.

73. Schenker JG. Etiology of and therapeutic approach to synechia uteri. Eur J Obstet Gynecol Reprod Biol 1996;65(1):109-113.

74. Shalev J, Meizner I, Bar-Hava Il. Predictive value of transvaginal sonography performed before routine diagnostic hysteroscopy for evaluation of infertility. Fertil Steril 2000;73:412.

75. Yu D, Wong YM, Cheong Y, Xia E, Li TC. Asherman syndromeone century later. Fertil Steril 2008;11:759-779.

76. Soares SR, Barbosa dos Reis MM, Camargos AF. Diagnostic accuracy of sonohysterography, transvaginal sonography, and hysterosalpingography in patients with uterine cavity diseases. Fertil Steril 2000;73(2):406-411.

77. AAGL Practice Report: Practice guidelines for management of intrauterine synechiae. J Minim Invas Gynecol 2010;17(1):1-7.

78. Kresowik JD, Syrop CH, Van Voorhis BJ, Ryan GL. Ultrasound is the optimal choice for guidance in difficult hysteroscopy. Ultrasound Obstet Gynecol 2012;11:715-718.

79. Schlaff WD, Hurst BS. Preoperative sonographic measurement of endometrial pattern predicts outcome of surgical repair in patients with severe Asherman's syndrome. Fertil Steril 1995;63(2):410-413.

80. The American Fertility Society classifications of adnexal adhesions, distal tubal occlusion secondary to tubal ligation, tubal pregnancies, Müllerian anomalies and intrauterine adhesions. Fertil Steril 1988;49(6):944-955.

81. Marcus S, al-Shawaf T, Brinsden P. The obstetric outcome of in vitro fertilization and embryo transfer in women with congenital uterine malformation. Am J Obstet Gynecol 1996;175(1):85-89.

82. Heinonen PK. Complete septate uterus with longitudinal vaginal septum. Fertil Steril 2006;85(3):700-705.
83. Speroff L, Glass RH, Kase NG. The uterus. In: Clinical Gynecologic Endocrinology and Infertility, Lippincott Williams \& Wilkins, Philadelphia 1999. p.148.

84. Baramki TA. Hysterosalpingography. Fertil Steril 2005; 83(6):1595-1606.

85. Ludwin A, Ludwin I, Banas T. Diagnostic accuracy of sonohysterography, hysterosalpingography and diagnostic hysteroscopy in diagnosis of arcuate, septate and bicornuate uterus. J Obstet Gynaecol Res 2011;37(3):178-186.

86. Ludwin A, Pitynski K, Ludwin I, Banas T, Knafel A. Two- and three-dimensional ultrasonography and sonohysterography versus hysteroscopy with laparoscopy in the differential diagnosis of septate, bicornuate, and arcuate uteri. J Minim Invasive Gynecol 2013;20(1):90-99.

87. Liu M, Wang SS, Lin XH, He SH. Clinical value of threedimensional transvaginal sonography in diagnosis of septate uterus and prediction of adverse pregnancy outcome. Zhonghua Fu Chan Ke Za Zhi 2013;48(7):499-503.

88. Fedele L, Bianchi S. Hysteroscopic metroplasty for septate uterus. Obstet Gynecol Clin North Am 1995;22(3):473-489.

89. Parker WH. Uterine myomas: management. Fertil Steril 2007;88(2):255-271.

90. Pritts EA, Parker WH, Olive DL. Fibroids and infertility: an updated systematic review of the evidence. Fertil Steril 2009;91(4):1215-1223.

91. Dimitrijevic D, Vasiljevic R, Anicic R, Brankovic S. Comparison of hysterosonography and hysteroscopy for diagnosing perimenopausal bleeding. Clin Exp Obstet Gynecol 2013; 40(2):246-249.

92. Fleischer AC, Shappell HW. Color Doppler sonohysterography of endometrial polyps and submucosal fibroids. J Ultrasound Med 2003;22(6):601-614.

93. Kurjak A, Kupesic S, Miric D. The assessment of benign uterine tumor vascularization by transvaginal color Doppler. Ultrasound Med Biol 1992;18:645-649.

94. Andrew $\mathrm{H}$, Critchley $\mathrm{H}$. The effect of uterine fibroids on embryo implantation. Semin Reprod Med 2007;25(6):483-489.

95. Tulandi T. Treatment of uterine fibroids: is surgery obsolete? $\mathrm{N}$ Engl J Med 2007;356(4):411-413.

96. Van Voorhis BJ. Outcomes from assisted reproductive technology. Obstet Gynecol 2006;107(1):183-200.

97. Lieng M, Istre O, Qvigstad E. Treatment of endometrial polyps: a systematic review. Acta Obstet Gynecol Scand 2010;89(8):992-1002.

98. Salim S, Won H, Nesbitt-Hawes E. Diagnosis and management of endometrial polyps: a critical review of the literature. J Minim Invasive Gynecol 2011;18(5):569-581.

99. Bhaduri M, Tomlinson G, Glanc P. Likelihood ratio of sonohysterographic findings for discriminating endometrial polyps from submucosal fibroids. J Ultrasound Med 2014;33(1): $22-25$.

100. Coughlan C, Ledger W, Wang Q, Liu F, Demirol A, Gurgan T, Cutting R, Ong K, Sallam H, Li TC. Recurrent implantation failure: definition and management. Reprod Biomed Online 2014;28(1):14-38.

101. Bosteels J, Kasius J, Weyers S. Hysteroscopy for treating subfertility associated with suspected major uterine cavity abnormalities. Cochrane Database Syst Rev 2013;1:CD009461. Available at: http://www.ncbi.nlm.nih.gov/pubmed/23440838. 


\section{SELF ASSESSMENT QUIZ}

1. A 29-year old-nulligravida presents to your clinic with no menses for the last 6 months after she discontinued her oral contraceptive. She also complains of painful intercourse and occasional 'night sweats'. Menarche was at age 13. She began taking the oral contraceptive at age 17 to regulate her menstrual cycles. On physical examination she is afebrile and in no acute distress. She is $170 \mathrm{~cm}$ tall and weigh $60 \mathrm{~kg}$. There is no acne, hirsuitism or galactorrhea. Pelvic examination reveals mildly atrophic mucosa. Pregnancy test is negative and serum prolactin levels are within normal limits. What is the next best step in the management of this patient?
A. Abdominal ultrasound
B. Repeat pregnancy test
C. Measurement of serum FSH
D. Measurement of serum LH
E. Tuberculin skin test

2. A 36-year-old nulligravida presents with complaints of menstrual irregularity for the past year and a half. She states her cycles were always regular since menarche at age 13 but have recently become prolonged and unpredictable. She and her husband have been trying to conceive for the past three years without success. On physical examination, she is afebrile and in no acute distress. Her vagina, cervix and uterus are within normal limits and no adnexal masses are noted. She began measuring her basal body temperature 6 months ago and noted it was biphasic, remaining elevated after day 14 . Serum progesterone levels are normal for luteal phase. What is the next best step in management?
A. Pregnancy test
B. Diagnostic laparoscopy
C. Measurement of serum FSH
D. Endometrial biopsy
E. Serial transvaginal ultrasound

3. A 40-year-old G3P1021 presents with chief complaint of menstrual irregularity for the past year. She states her periods were regular since menarche at age 11 but they have become much shorter, occurring every 22 days. Her obstetric history is significant for 2 spontaneous abortions at 10 and 8 weeks gestation. She denies any physical complaints. She is married for five years and has one child (age 3). On physical examination, she is afebrile and in no acute distress. Her pelvic examination was within normal limits. Today is the 20th day of her menstrual cycle and you decide to obtain a serum progesterone level and pelvic ultrasound. Knowing that the average resistive index (RI) values during follicular phase are $0.56 \pm 0.06$, which of the following sets of values are expected in this patient?

A. RI $0.56 \pm 0.04$; progesterone $24.8 \mathrm{ng} / \mathrm{ml}$

B. RI $0.56 \pm 0.04$; progesterone $2.0 \mathrm{ng} / \mathrm{ml}$

C. RI $0.44 \pm 0.04$; progesterone $24.8 \mathrm{ng} / \mathrm{ml}$

D. RI $0.44 \pm 0.04$; progesterone $2.0 \mathrm{ng} / \mathrm{ml}$

4. A 24-year-old nulligravida presents to your clinic with chief complaint of infertility. Her past medical history is significant for a Chlamydia infection when she was 16 and an episode of PID last year. She complains of a constant, dull pelvic pain and a sensation of 'fullness'. Menarche was at 11 and has always been regular. She took oral contraceptives for three years starting at age 19. Vital signs are within normal limits. Pelvic examination is significant for bilateral adnexal masses. Pelvic ultrasound will demonstrate:

A. Thick tubal walls and free fluid in the abdomen

B. Thickened endometrium with discrete vascularization at the periphery

C. Homogenous 'carpet-like density at the level of the ovaries

D. Fluid filled, thin tubal walls and incomplete septations

5. A 20-year-old obese female presents to your clinic with menstrual irregularity for the past 5 months. She also complains of weight gain over the past year despite changes in her diet. Family history is significant for endometrial cancer in her mother and aunt. She does not use tobacco, alcohol, or drugs. BMI is $31.2 \mathrm{~kg} / \mathrm{m}^{2}$. On physical examination you note acne and dark facial hair. Serum prolactin and thyroid levels are normal. Urine pregnancy test is negative. Which of the following tests is indicated at this time?
A. Oral glucose tolerance test
B. Diagnostic laparoscopy
C. Trial of glucocorticoids
D. $A$ and $C$
E. All of the above

6. A 31-year-old nulligravida presents to the clinic for infertility evaluation. She has chronic pelvic pain that she rates $4 / 10$. She notes an increase in pain during menses and intercourse. Menarche was at age 12 and has always been regular. She is in a monogamous relationship with her husband and has no history of sexually transmitted diseases. BMI is $23.2 \mathrm{~kg} / \mathrm{m}^{2}$. Pelvic examination is significant for enlarged left adnexa. Transvaginal ultrasound demonstrates a thick-walled cyst with a homogenous 'ground-glass' appearance. What is the most likely diagnosis?
A. Teratoma
B. Cystadenocarcinoma
C. Endometrioma
D. Leiomyoma
E. Endometrial polyp

7. A 32-year-old primary infertile female presents to your clinic with chief complaint of infertility. She notes spotting in between menstrual cycles but has no other symptoms. Menarche was at age 10 and has been regular up until 3 months ago. She is sexually active with her husband and is not taking an oral contraceptive. Medical history is significant for mild hypertension for which she takes hydrochlorothiazide. There are no abnormalities on physical examination. BMI is $28.2 \mathrm{~kg}$ / $\mathbf{m}^{2}$. Pelvic ultrasound demonstrates focal thickening of the endometrium. The addition of color Doppler shows a single feeding vessel. What is the next best step in management of this woman's infertility?
A. Oral contraceptives
B. Hysteroscopic resection
C. Exploratory laparotomy
D. Expectant management
E. Hysterectomy 
8. A 28-year-old G3P1021 presents to the clinic for 'not having my period in 5 months'. She also reports mild intermittent pelvic pain. Menarche was at age 12 and has always been regular up until 5 months ago. She is sexually active with three partners and uses condoms 'most of the time'. A home pregnancy test was negative. She underwent 2 elective abortions, the most recent 6 months ago. You decide to perform pelvic ultrasound and find dense, hyperechogenic bands and are unable to delineate the endometrium. What is the most likely diagnosis?
A. Intrauterine adhesions
B. Endometrial polyp
C. Submucosal fibroid
D. Premature ovarian failure
E. Endometrial cancer

9. A 22-year-old G4P0040 presents to the clinic with chief complaint of recurrent pregnancy loss. She has been pregnant 3 times in the past 2 years and lost every pregnancy before the second trimester. Menarche was at age 13 and occurs at regular intervals. She notes significant pain and cramping during her menstrual periods. Family history is significant for a 'heart-shaped' uterus in one of her aunts. You suspect a congenital
Mullerian anomaly. What is most appropriate and costeffective imaging modality for this patient?
A. Fluoroscopy
B. Transabdominal ultrasound
C. CT scan
D. 3D ultrasound
E. MRI

10. A 34-year-old G2P1011 presents to your office with chief complaint of heavy and prolonged menstrual periods. She also notes exertional fatigue. Menarche was at age 12 and was always regular up until a year ago when it began lasting 10 to 12 days each month. She had a miscarriage 7 months ago at 12 weeks gestation. Past medical history and family history are insignificant. On physical examination, the uterus is diffusely enlarged and no adnexal masses are palpated. Laboratory values are significant for hemoglobin of $8.8 \mathrm{gm} / \mathrm{dl}$ and hematocrit of $24 \%$. You perform saline infusion sonohysterography (SIS) and see a round, calcified mass protruding into the endometrium. What is the mechanism of calcification in this lesion?
A. Amyloid deposits
B. Ischemia followed by calcium accumulation
C. Hyperplastic endometrial cells
D. Excessive nutrient metabolism
E. Malignant degeneration

Correct Answers:
1. C;
2. E;
3. B;
4. D;
5. A;
6. C;
7. B;
8. A;
9. D;
10. B 